Article

\title{
Fossil Genera in Elateridae (Insecta, Coleoptera): A Triassic Origin and Jurassic Diversification
}

\author{
Robin Kundrata *(D), Gabriela Packova and Johana Hoffmannova \\ Department of Zoology, Faculty of Science, Palacky University, 17. listopadu 50, 77146 Olomouc, \\ Czech Republic; gabriela.packova01@upol.cz (G.P.); johana.hoffmannova01@upol.cz (J.H.) \\ * Correspondence: robin.kundrata@upol.cz
}

Received: 2 June 2020; Accepted: 23 June 2020; Published: 26 June 2020

\begin{abstract}
Insect fossils bear important information about the evolutionary history of the group. The fossil record of Elateridae, a large cosmopolitan beetle family, has been greatly understudied and the available data are often replete with ambiguity and uncertainty. The research of Elateridae evolution cannot be done without solid genus-group name concepts. In this study we provide an updated comprehensive summary of the fossil genera in Elateridae, including their systematic placement and information on the type species, gender, number of species, age range, and relevant bibliography. We list seven valid fossil genera in Agrypninae, one in Cardiophorinae, two in Dendrometrinae, five in Elaterinae, two in Negastriinae, one in Omalisinae, one in Pityobiinae, and 36 in Protagrypninae. Additional 19 genera are tentatively classified as Elateridae incertae sedis, and their placements are discussed. Further, we move genera Babuskaya Martins-Neto \& Gallego, 2009, Cardiosyne Martins-Neto \& Gallego, 2006, Fengningia Hong, 1984 and Gemelina Martins-Neto \& Gallego, 2006 from Elateridae to Coleoptera incertae sedis. We also discuss the genera previously placed in Elateridae, which are currently not included in the family. The data on the fossil generic diversity suggest that Elateridae originated in the Triassic and rapidly diversified and became comparatively abundant through the Jurassic. We call for further research on the fossil Elateridae from various deposits in order to increase our knowledge on the origin, evolution, and palaeodiversity of the group.
\end{abstract}

Keywords: classification; Cenozoic; click-beetles; Elateroidea; evolution; Mesozoic; palaeodiversity; systematics

\section{Introduction}

Elateridae, or click-beetles, is a large beetle family containing about 10,000 described species worldwide [1]. This group includes several ecologically and economically important lineages, with some of them being among the most serious agricultural pests [2]. The current composition of Elateridae considerably differs from the historical concepts of the group, which usually included only hard-bodied lineages with a well-developed clicking mechanism, consisting of a prosternal process fitting into a mesoventral cavity [3-6]. With the rise of molecular phylogenetic analyses, several soft-bodied groups, which were previously considered separate families, i.e., Drilidae, Omalisidae, and Plastoceridae, were merged with Elateridae [7,8]. However, the internal phylogenetic relationships, as well as the suprageneric classification of the group, remain open to further study [9-11].

Elateridae have a long evolutionary history which dates back to the early Mesozoic [12-17]. Numerous taxa were reported mainly from the Jurassic deposits of China, Kazakhstan, and Kyrgyzstan [13,17-22], but the rich elaterid fossil record is known also from various Cretaceous sedimentary deposits and amber [16,23-28]. The Cenozoic Elateridae fossils are known mainly from the Eocene deposits of the United States and Europe [29-31], and from the Baltic amber [32]. Altogether, the fossil record of Elateridae includes approximately 300 species classified in more than 100 genera [28,33]. Regarding 
the fossil genera in Elateridae, Scudder [34] published the World catalogue of the fossil insects which included also taxa assigned to this family. Handlirsch [35] listed in Elateridae many doubtful taxa which can belong to various other beetle families. Hyslop [36] was the first who attempt to cover all genus-group names in Elateridae. He also catalogued the fossil genera, including those previously listed by Handlirsch [35]. Dolin [13] revised the fossil record of Elateridae from the Jurassic of Karatau (Karabastau Formation of Kazakhstan). Spahr [37] published the catalogue of beetles from amber and copal. Carpenter [38] compiled the list of fossil genera in Coleoptera, including Elateridae, and placed many dubious taxa into Coleoptera incertae sedis. Alekseev [39] provided the checklist of beetles described from the Baltic amber. The online open checklist of fossil beetles by Kirejtshuk and Ponomarenko [33] includes also Elateridae but requires some updates, including changes in classification. Most recently, the first part of the World catalogue of the genus-group names in Elateridae contained only extant taxa [40].

Fossils represent an important data source for understanding morphological character evolution, elucidating the relationships among lineages, and dating the phylogenetic divergence events. However, it is crucial to understand the taxonomic and phylogenetic position of fossil taxa in order to correctly interpret the evolutionary history of the group. Consequently, misinterpretations and misidentifications of fossils often result in incorrect conclusions $[15,16]$. Such research cannot be carried out without the solid genus-group name concepts which are consistent with regulations from the International Commission on Zoological Nomenclature [41]. In this study, we provide a summary of all fossil genus-group names including type species and their designations, misspellings, correct gender, and their systematic position according to the most recent publications. This study could serve as a starting point for future research on the click-beetle palaeodiversity and evolution.

\section{Materials and Methods}

We provide information on all fossil genus-group names in Elateridae, including their current systematic status. The family classification follows that of Kundrata et al. [9], with subsequent changes made by Kusy et al. [8] and Kundrata et al. [10]. Generally, we follow the style used in the first part of the World catalogue of the genus-group names in Elateridae [40]. The names of the family-, genus- and (type) species-group taxa are given with the name of the author, and the year and page of publication. The page given is the page where the taxon name and description are printed. The year and page given for the incorrect subsequent spellings are the first year and page in which they are used. Incorrect subsequent spellings not in prevailing usage are unavailable according to Art. 33.3 of the Code [41]. We provide the type species for each genus-group name, including information on its designation. Misspellings are followed by colon ":". Taxa marked with an asterisk (*) contain also recent species. Only fossil genera are listed, except for the recent Athous Eschscholtz, 1829, Ampedus Dejean, 1833 and Limonius Eschscholtz, 1829, each of which contains one fossil subgenus. The age of fossils was taken from the Paleobiology Database (https://paleobiodb.org/). Divisions of geological time and their boundaries follow the ICS International Chronostratigraphic Chart v. 2020/01 (http://www.stratigraphy.org/) [42]. The overview of the fossil genera and subgenera in Elateridae is summarized in Table 1. 
Table 1. Overview of the fossil genera and subgenera in Elateridae.

\begin{tabular}{|c|c|c|c|c|}
\hline Subfamily Tribe & Genus & Period/Epoch, Location & Age (Ma) & Nr. of Species \\
\hline \multicolumn{5}{|l|}{ Agrypninae } \\
\hline \multirow[t]{5}{*}{ Agrypnini } & Ageratus Dolin, 1980 & Jurassic of Kazakhstan & $166.1-157.3$ & 2 \\
\hline & Compsoderus Dolin, 1980 & Jurassic of Kazakhstan & $166.1-157.3$ & 1 \\
\hline & Litholacon Dolin, 1980 & Jurassic of Kazakhstan & $166.1-157.3$ & 7 \\
\hline & Macropunctum Tröster, 1991 & $\begin{array}{l}\text { Eocene of Germany and } \\
\text { United Kingdom }\end{array}$ & $48.6-33.9$ & 13 \\
\hline & Plagioraphes Iablokoff-Khnzorian, 1961 & Eocene Baltic amber & $38.0-33.9$ & 1 \\
\hline Cryptocardiini & Cryptocardius Dolin, 1980 & Jurassic of Kazakhstan & $166.1-157.3$ & 1 \\
\hline Pyrophorini & Eopyrophorus Haupt, 1950 & Eocene of Germany & $47.8-41.3$ & 1 \\
\hline \multicolumn{5}{|l|}{ Cardiophorinae } \\
\hline & Mionelater Becker, 1963 & Miocene of Mexico & $23.0-16.0$ & 1 \\
\hline \multicolumn{5}{|l|}{ Dendrometrinae } \\
\hline \multirow[t]{2}{*}{ Dendrometrini } & Athousiomorphus Iablokoff-Khnzorian, 1961 * & Eocene Baltic amber & $38.0-33.9$ & 1 \\
\hline & Paralimonius Iablokoff-Khnzorian, 1961 ** & Eocene Baltic amber & $38.0-33.9$ & 1 \\
\hline Dimini & Alaodima Dolin, 1980 & Jurassic of Kazakhstan & $166.1-157.3$ & 1 \\
\hline Hypnoidini & Cryptagriotes Wickham, 1916 & Eocene of the United States & $37.2-33.9$ & 1 \\
\hline \multicolumn{5}{|l|}{ Elaterinae } \\
\hline Ampedini & Octamenogonoides Iablokoff-Khnzorian, 1961 *** & Eocene Baltic amber & $38.0-33.9$ & 1 \\
\hline \multirow[t]{4}{*}{ Elaterini } & Diaraphes Iablokoff-Khnzorian, 1961 & Eocene Baltic amber & $38.0-33.9$ & 1 \\
\hline & Elatron Iablokoff-Khnzorian, 1961 & Eocene Baltic amber & $38.0-33.9$ & 1 \\
\hline & Holopleurus Iablokoff-Khnzorian, 1961 & Eocene Baltic amber & $38.0-33.9$ & 1 \\
\hline & Orthoraphes Iablokoff-Khnzorian, 1961 & Eocene Baltic amber & $38.0-33.9$ & 1 \\
\hline incertae sedis & Crioraphes Iablokoff-Khnzorian, 1961 & Eocene Baltic amber & $38.0-33.9$ & 1 \\
\hline \multicolumn{5}{|l|}{ Negastriinae } \\
\hline & Ganestrius Dolin, 1976 & Jurassic of Kazakhstan & $166.1-157.3$ & 2 \\
\hline & Protoquasimus Dolin, 1976 & Jurassic of Kazakhstan & $166.1-157.3$ & 1 \\
\hline \multicolumn{5}{|l|}{ Omalisinae } \\
\hline & Jantarokrama Kirejtshuk \& Kovalev, 2015 & Eocene Baltic amber & $38.0-33.9$ & 1 \\
\hline \multicolumn{5}{|l|}{ Pityobiinae } \\
\hline & Cretopityobius Otto, 2019 & Cretaceous of Myanmar & $99.6-93.5$ & 1 \\
\hline \multicolumn{5}{|l|}{ Protagrypninae } \\
\hline \multirow[t]{6}{*}{ Desmatini } & Anoixis Chang, Kirejtshuk \& Ren, 2010 & Cretaceous of China & $125.5-122.5$ & 1 \\
\hline & Apoclion Chang, Kirejtshuk \& Ren, 2010 & Cretaceous of China & $125.5-122.5$ & 3 \\
\hline & Desmatinus Chang, Kirejtshuk \& Ren, 2010 & Cretaceous of China & $125.5-122.5$ & 1 \\
\hline & Desmatus Dolin, 1975 & Jurassic of Kazakhstan & $166.1-157.3$ & 4 \\
\hline & $\begin{array}{l}\text { Paradesmatus Chang, Kirejtshuk \& Ren in } \\
\text { Chang et al., } 2009\end{array}$ & $\begin{array}{l}\text { Jurassic and Cretaceous of } \\
\text { China }\end{array}$ & $166.1-122.5$ & 3 \\
\hline & Plesiorhaphes Dolin, 1980 & Jurassic of Kazakhstan & $166.1-157.3$ & 1 \\
\hline \multirow[t]{12}{*}{ Hypnomorphini } & Abrotus Dolin, 1980 & Jurassic of Kazakhstan & $166.1-157.3$ & 2 \\
\hline & Adiagnostus Dolin, 1980 & Jurassic of Kazakhstan & $166.1-157.3$ & 3 \\
\hline & Codemus Dolin, 1980 & Jurassic of Kazakhstan & $166.1-157.3$ & 10 \\
\hline & Elaterophanes Handlirsch, 1906 & $\begin{array}{l}\text { Triassic and Jurassic of the } \\
\text { United Kingdom }\end{array}$ & $208.5-189.6$ & 3 \\
\hline & Graciolacon Dolin, 1980 & Jurassic of Kazakhstan & $166.1-157.3$ & 1 \\
\hline & Hypnomorphoides Dolin, 1980 & Jurassic of Kazakhstan & $166.1-157.3$ & 4 \\
\hline & Hypnomorphus Dolin, 1975 & Jurassic of Kazakhstan & $166.1-157.3$ & 14 \\
\hline & Idiomorphus Dolin, 1980 & Jurassic of Kazakhstan & $166.1-157.3$ & 2 \\
\hline & Lapidiconides Dolin, 1980 & Jurassic of Kazakhstan & $166.1-157.3$ & 3 \\
\hline & Lapidostenus Dolin, 1980 & Jurassic of Kazakhstan & $166.1-157.3$ & 5 \\
\hline & Lithoptychus Dolin, 1980 & Jurassic of Kazakhstan & $166.1-157.3$ & 4 \\
\hline & Lithosomus Dolin, 1980 & Jurassic of Kazakhstan & $166.1-157.3$ & 2 \\
\hline
\end{tabular}


Table 1. Cont

\begin{tabular}{|c|c|c|c|c|}
\hline Subfamily Tribe & Genus & Period/Epoch, Location & Age (Ma) & Nr. of Species \\
\hline \multicolumn{5}{|l|}{ Protagrypninae } \\
\hline \multirow[t]{4}{*}{ Hypnomorphini } & Necrocoelus Dolin, 1980 & Jurassic of Kazakhstan & $166.1-157.3$ & 1 \\
\hline & Negastrioides Dolin, 1980 & Jurassic of Kazakhstan & $166.1-157.3$ & 4 \\
\hline & Parahypnomorphus Dolin, 1980 & Jurassic of Kazakhstan & $166.1-157.3$ & 3 \\
\hline & Platyelater Dolin, 1980 & Jurassic of Kazakhstan & $166.1-157.3$ & 4 \\
\hline Pollostelaterini & Pollostelater Alekseev, 2011 & $\begin{array}{l}\text { Cretaceous of Russian } \\
\text { Federation }\end{array}$ & $125.0-113.0$ & 1 \\
\hline \multirow[t]{12}{*}{ Protagrypnini } & Acheonus Dolin, 1980 & Jurassic of Kazakhstan & $166.1-157.3$ & 3 \\
\hline & Archaeolus Lin, 1986 & Jurassic of China & $170.3-168.3$ & 1 \\
\hline & Clavelater Dong \& Huang, 2011 & Jurassic of China & $166.1-157.3$ & 1 \\
\hline & Koreagrypnus Sohn \& Nam in Sohn et al., 2019 & Cretaceous of South Korea & $113.0-100.5$ & 1 \\
\hline & Lithocoelus Dolin, 1975 & Jurassic of Kazakhstan & $166.1-157.3$ & 2 \\
\hline & Lithomerus Dolin, 1980 & $\begin{array}{l}\text { Jurassic of Australia and } \\
\text { Kazakhstan, Cretaceous of } \\
\text { China }\end{array}$ & $182.7-122.5$ & 6 \\
\hline & Megalithomerus Sohn \& Nam in Sohn et al., 2019 & Cretaceous of South Korea & $113.0-100.5$ & 1 \\
\hline & Micragrypnites Dolin, 1973 & Jurassic of Kyrgyzstan & $201.3-190.8$ & 1 \\
\hline & Paragrypnites Dolin, 1980 & Jurassic of Kazakhstan & $166.1-157.3$ & 1 \\
\hline & Paraprotagrypnus Chang, Zhao \& Ren, 2009 & Jurassic of China & $166.1-157.3$ & 1 \\
\hline & Protagrypnus Dolin, 1973 & $\begin{array}{l}\text { Jurassic of Kyrgyzstan and } \\
\text { China }\end{array}$ & 201.3-157.3 & 2 \\
\hline & Sinolithomerus Dong \& Huang, 2009 & Jurassic of China & $166.1-157.3$ & 1 \\
\hline incertae sedis & Paralithomerus Chang, Zhang \& Ren, 2008 & Cretaceous of China & $125.5-122.5$ & 2 \\
\hline \multicolumn{5}{|l|}{$\begin{array}{l}\text { Elateridae incertae } \\
\text { sedis }\end{array}$} \\
\hline & Adocetus Scudder, 1900 & Eocene of the United States & $55.8-50.3$ & 1 \\
\hline & Artinama Lin, 1986 & Jurassic of China & $199.3-190.8$ & 1 \\
\hline & Bilineariselater Chang \& Ren, 2008 & Cretaceous of China & $125.5-122.5$ & 1 \\
\hline & Cretoelaterium Alekseev, 2008 & $\begin{array}{l}\text { Cretaceous of Russian } \\
\text { Federation }\end{array}$ & $129.4-125.0$ & 1 \\
\hline & Cryptocoelus Dolin \& Nel, 2002 & $\begin{array}{l}\text { Cretaceous of China and } \\
\text { Russian Federation }\end{array}$ & $125.5-113.0$ & 8 \\
\hline & Curtelater Chang \& Ren, 2008 & Cretaceous of China & $125.5-122.5$ & 1 \\
\hline & Elateridium Tillyard, 1918 & Triassic of Australia & $247.2-208.5$ & 3 \\
\hline & Elaterites Heer, 1847 & $\begin{array}{l}\text { Paleocene of Argentina, } \\
\text { Eocene of the United } \\
\text { Kingdom, Germany and } \\
\text { Czech Republic, Oligocene } \\
\text { of Switzerland, Miocene of } \\
\text { Germany }\end{array}$ & $66.0-11.6$ & 12 \\
\hline & Elaterium Westwood, 1854 & $\begin{array}{l}\text { Triassic of Australia, } \\
\text { Cretaceous of the United } \\
\text { Kingdom }\end{array}$ & $228.0-140.2$ & 2 \\
\hline & Gripecolous Lin, 1986 & Jurassic of China & $170.3-168.3$ & 1 \\
\hline & Ludiophanes Wickham, 1916 & Eocene of the United States & $38.0-33.9$ & 1 \\
\hline & Mercata Lin, 1986 & Jurassic of China & $170.3-168.3$ & 1 \\
\hline & Ovivagina Zhang, 1997 & Jurassic of China & 201.3-190.8 & 1 \\
\hline & Protocardiophorus Dolin, 1976 & Jurassic of Kazakhstan & $166.1-157.3$ & 2 \\
\hline & Pseudocardiophorites Dolin, 1976 & Jurassic of Kazakhstan & $166.1-157.3$ & 5 \\
\hline & Silicernius Heyden, 1859 & Oligocene of Germany & $28.4-23.0$ & 1 \\
\hline & Sinoelaterium Ping, 1928 & Cretaceous of China & $125.5-122.5$ & 1 \\
\hline & Tetraraphes Iablokoff-Khnzorian, 1961 & Eocene Baltic amber & $38.0-33.9$ & 1 \\
\hline & Turonelater Alekseev, 2011 & Cretaceous of Kazakhstan & $93.9-89.8$ & 1 \\
\hline
\end{tabular}

* Subgenus of Athous Eschscholtz, 1829, ** Subgenus of Limonius Eschscholtz, 1829, *** Subgenus of Ampedus

Dejean, 1833. 


\section{Results}

Family Elateridae Leach, 1815*

Elaterides Leach, 1815: 85 [43]. Type genus: Elater Linnaeus, 1758. For more information, including synonyms, see Bouchard et al. [44] and Kundrata et al. [40].

\subsection{Overview of the Fossil Genera and Subgenera in Elateridae}

\subsubsection{Subfamily Agrypninae Candèze, $1857^{*}$}

Agrypnides Candèze, 1857: 17 [45]. Type genus: Agrypnus Eschscholtz, 1829. For more information, including synonyms, see Kundrata et al. [40].

- $\quad$ Tribe Agrypnini Candèze, $1857^{*}$

Agrypnides Candèze, 1857: 17 [45]. Type genus: Agrypnus Eschscholtz, 1829. For more information, including synonyms, see Kundrata et al. [40].

Genus Ageratus Dolin, 1980

Ageratus Dolin, 1980: 72 [13]. Gender: masculine. Type species: Ageratus ponomarenkoi Dolin, 1980: 73 [13]; by original designation. Jurassic of Kazakhstan (166.1-157.3 Ma). Two species.

Literature. Dolin (1980: 72) [13], Carpenter (1992: 304) [38], Korneev \& Cate (2005: 9) [46], Alekseev (2011: 424) [26].

Genus Compsoderus Dolin, 1980

Compsoderus Dolin, 1980: 71 [13]. Gender: masculine. Type species: Compsoderus priscus Dolin, 1980: 72 [13]; by original designation. Jurassic of Kazakhstan (166.1-157.3 Ma). Monotypic.

Compsoferus: Carpenter, 1992: 304 [38]; unavailable name, incorrect subsequent spelling not in prevailing usage [41].

Literature. Dolin (1980: 71) [13], Carpenter (1992: 304) [38], Korneev \& Cate (2005: 10) [46].

Genus Litholacon Dolin, 1980

Litholacon Dolin, 1980: 67 [13]. Gender: masculine. Type species: Litholacon derumpens Dolin, 1980: 68 [13]; by original designation. Jurassic of Kazakhstan (166.1-157.3 Ma). Seven species.

Literature. Dolin (1980: 67) [13], Carpenter (1992: 305) [38], Korneev \& Cate (2005: 10) [46], Alekseev (2011: 424) [26].

Genus Macropunctum Tröster, 1991

Macropunctum Tröster, 1991: 100 [31]. Gender: neuter. Type species: Macropunctum messelense Tröster, 1991: 106 [31]; by original designation. Eocene of Germany (48.6-40.4 Ma), Eocene of the United Kingdom (38.0-33.9 Ma). 13 species.

Literature. Tröster (1991: 100) [31], Tröster (1992: 111) [47], Tröster (1993: 49) [48], Tröster (1994a: 58) [49], Tröster (1994b: 145) [50], Tröster (1999: 12) [51], Wappler (2003: 86) [52], Kirejtshuk et al. (2019: 453) [53].

Genus Plagioraphes Iablokoff-Khnzorian, 1961

Plagioraphes Iablokoff-Khnzorian, 1961: 84 [32]. Gender: masculine. Type species: Plagioraphes fasciatus Iablokoff-Khnzorian, 1961: 85 [32]; by original designation. Eocene Baltic amber (38.0-33.9 Ma). Monotypic.

Literature. Iablokoff-Khnzorian (1961: 84) [32], Larsson (1978: 153) [54], Spahr (1981: 49) [37], Carpenter (1992: 305) [38], Alekseev (2013: 7) [39], Alekseev (2017: 409) [55].

Remark. This genus was originally placed in Agrypninae [32]. Alekseev [39] listed Plagioraphes under Pityobiinae but without any justification or clarification. Here, we follow the original placement by Iablokoff-Khnzorian [32]. 
- Tribe Cryptocardiini Dolin, 1980

Cryptocardiini Dolin, 1980: 74 [13]. Type genus: Cryptocardius Dolin, 1980.

Genus Cryptocardius Dolin, 1980

Cryptocardius Dolin, 1980: 74 [13]. Gender: masculine. Type species: Cryptocardius mirabilis Dolin, 1980: 75 [13]; by original designation. Jurassic of Kazakhstan (166.1-157.3 Ma). Monotypic.

Literature. Dolin (1980: 74) [13], Carpenter (1992: 304) [38], Korneev \& Cate (2005: 10) [46], Chang et al., (2007: 1248) [56], Alekseev (2011: 423) [26].

- $\quad$ Tribe Pyrophorini Candèze, $1863^{*}$

Pyrophorites Candèze, 1863: 3 [57]. Type genus: Pyrophorus Billberg, 1820.

Genus Eopyrophorus Haupt, 1950

Eopyrophorus Haupt, 1950: 101 [30]. Gender: masculine. Type species: Eopyrophorus mixtus Haupt, 1950: 107 [30]; by monotypy. Eocene of Germany (47.8-41.3 Ma). Monotypic.

Literature. Haupt (1950: 101) [30], Haupt (1956: 48) [58], Kinzelbach \& Lutz (1985: 600) [59], Carpenter (1992: 304) [38].

\subsubsection{Subfamily Cardiophorinae Candèze, $1859^{*}$}

Cardiophorites Candèze, 1859: 4 [60]. Type genus: Cardiophorus Eschscholtz, 1829. For more information, including synonyms, see Bouchard et al. [44].

Genus Mionelater Becker, 1963

Mionelater Becker, 1963: 125 [61]. Gender: masculine. Type species: Mionelater planatus Becker, 1963: 126 [61]; by original designation. Miocene of Mexico (Chiapas amber) (23.0-16.0 Ma). Monotypic.

Literature. Becker (1963: 125) [61], Spahr (1981: 49) [37], Carpenter (1992: 305) [38], Solórzano Kraemer (2007: 120) [62], Douglas (2017: 50) [63].

Remark. Douglas [63] suggested that based on the shapes of head, posterior pronotal angles and mesocoxal cavities, this genus might belong to a different subfamily.

\subsubsection{Subfamily Dendrometrinae Gistel, $1848^{*}$}

Dendrometridae Gistel, 1848: 5 [64]. Type genus: Dendrometrus Gistel, 1848. For more information, including synonyms, see Bouchard et al. [44].

- $\quad$ Tribe Dendrometrini Reitter, $1905^{*}$

Dendrometridae Gistel, 1848: 5 [64]. Type genus: Dendrometrus Gistel, 1848. For more information, including synonyms, see Bouchard et al. [44].

Genus Athous Eschscholtz, 1829*

Athous Eschscholtz, 1829: 33 [65]. Type species: Elater vittatus Fabricius, 1792: 224 [66]; by subsequent designation (Westwood 1838: 26) [67]. For more information, including synonyms, see Sánchez-Ruiz [68] and Cate [69]. 
Subgenus Athousiomorphus Iablokoff-Khnzorian, 1961

Athousiomorphus Iablokoff-Khnzorian, 1961: 92 [32]. Gender: masculine. Type species: Athous (Athousiomorphus) olgae Iablokoff-Khnzorian, 1961: 92 [32]; by original designation. Eocene Baltic amber (38.0-33.9 Ma). Monotypic.

Athousimorphus: Alekseev, 2013: 7 [39]; unavailable name, incorrect subsequent spelling not in prevailing usage [41].

Literature. Iablokoff-Khnzorian (1961: 92) [32], Larsson (1978: 153) [54], Spahr (1981: 46) [37], Alekseev (2013: 7) [39].

Genus Limonius Eschscholtz, 1829*

Limonius Eschscholtz, 1829: 33 [65]. Type species: Elater minutus Linnaeus, 1758: 406 [70]; by subsequent designation (Curtis 1838: 694) [71]. For more information, including synonyms, see Sánchez-Ruiz [68] and Cate [69].

Subgenus Paralimonius Iablokoff-Khnzorian, 1961

Paralimonius Iablokoff-Khnzorian, 1961: 91 [32]. Gender: masculine. Type species: Limonius (Paralimonius) barovskyi Iablokoff-Khnzorian, 1961: 91 [32]; by original designation. Eocene Baltic amber (38.0-33.9 Ma). Monotypic.

Literature. Iablokoff-Khnzorian (1961: 91) [32], Larsson (1978: 153) [54], Spahr (1981: 48) [37], Alekseev (2013: 7) [39].

- $\quad$ Tribe Dimini Candèze, $1863^{*}$

Dimites Candèze, 1863: 237 [57]. Type genus: Dima Charpentier, 1825.

Genus Alaodima Dolin, 1980

Alaodima Dolin, 1980: 75 [13]. Gender: feminine. Type species: Alaodima grandis Dolin, 1980: 76 [13]; by original designation. Jurassic of Kazakhstan (166.1-157.3 Ma). Monotypic.

Literature. Dolin (1980: 75) [13], Carpenter (1992: 304) [38], Korneev \& Cate (2005: 9) [46], Kundrata et al., (2018: 68) [72].

- Tribe Hypnoidini Schwarz, $1906^{*}$

Hypnoidini Schwarz, 1906: 150 [3]. Type genus: Hypnoidus Dillwyn, 1829.

Genus Cryptagriotes Wickham, 1916

Cryptagriotes Wickham, 1916: 512 [29]. Gender: masculine. Type species: Cryptagriotes minusculus Wickham, 1916: 512 [29]; by original designation. Eocene of the United States (37.2-33.9 Ma). Monotypic.

Literature. Wickham (1916: 512) [29], Hyslop (1921: 637) [36], Carpenter (1992: 304) [38].

\subsubsection{Subfamily Elaterinae Leach, $1815^{*}$}

Elaterides Leach, 1815: 85 [43]. Type genus: Elater Linnaeus, 1758. For more information, including synonyms, see Bouchard et al. [44].

- $\quad$ Tribe Ampedini Fleutiaux, $1947^{*}$

Ampedidae Gistel, 1848: 5 [64]. Type genus: Ampedus Dejean, 1833. 
Genus Ampedus Dejean, 1833*

Ampedus Dejean, 1833: 92 [73]. Type species: Elater sanguineus Linnaeus, 1758: 405 [70]; by subsequent designation (Curtis 1838: 694) [71]. For more information, including synonyms, see Sánchez-Ruiz [68] and Cate [69].

Subgenus Octamenogonoides Iablokoff-Khnzorian, 1961

Octamenogonoides Iablokoff-Khnzorian, 1961: 88 [32]. Gender: masculine. Type species: Elater (Octamenogonoides) gebleri Iablokoff-Khnzorian, 1961: 88 [32]; by original designation. Eocene Baltic amber (38.0-33.9 Ma). Monotypic.

Octamenagonoides: Alekseev, 2013: 7 [39]; unavailable name, incorrect subsequent spelling not in prevailing usage [41].

Literature. Iablokoff-Khnzorian (1961: 88) [32], Spahr (1981: 48) [37], Alekseev (2013: 7) [39].

Remark. It was originally described as a subgenus of Elater Linnaeus, 1758 [32]. Alekseev [39] transferred Octamenogonoides to Ampedus Dejean, 1833.

- $\quad$ Tribe Elaterini Leach, $1815^{*}$

Elaterides Leach, 1815: 85 [43]. Type genus: Elater Linnaeus, 1758. For more information, including synonyms, see Bouchard et al. [44].

Genus Diaraphes Iablokoff-Khnzorian, 1961

Diaraphes Iablokoff-Khnzorian, 1961: 89 [32]. Gender: masculine. Type species: Diaraphes kozhantshikovi Iablokoff-Khnzorian, 1961: 89 [32]; by original designation. Eocene Baltic amber (38.0-33.9 Ma). Monotypic.

Literature. Iablokoff-Khnzorian (1961: 89) [32], Larsson (1978: 153) [54], Spahr (1981: 47) [37], Carpenter (1992: 304) [38], Alekseev (2013: 7) [39], Alekseev (2017: 409) [55].

Genus Elatron Iablokoff-Khnzorian, 1961

Elatron Iablokoff-Khnzorian, 1961: 90 [32]. Gender: neuter. Type species: Elatron semenovi Iablokoff-Khnzorian, 1961: 90 [32]; by original designation. Eocene Baltic amber (38.0-33.9 Ma). Monotypic.

Literature. Iablokoff-Khnzorian (1961: 90) [32], Larsson (1978: 153) [54], Spahr (1981: 48) [37], Carpenter (1992: 304) [38], Alekseev (2013: 7) [39], Alekseev (2017: 409) [55].

Genus Holopleurus Iablokoff-Khnzorian, 1961

Holopleurus Iablokoff-Khnzorian, 1961: 86 [32]. Gender: masculine. Type species: Holopleurus succineus Iablokoff-Khnzorian, 1961: 86 [32]; by original designation. Eocene Baltic amber (38.0-33.9 Ma). Monotypic.

Holopeurus: Carpenter, 1992: 304 [38]; unavailable name, incorrect subsequent spelling not in prevailing usage [41].

Literature. Iablokoff-Khnzorian (1961: 86) [32], Larsson (1978: 153) [54], Spahr (1981: 48) [37], Carpenter (1992: 304) [38], Alekseev (2013: 7) [39], Alekseev (2017: 409) [55].

Genus Orthoraphes Iablokoff-Khnzorian, 1961

Orthoraphes Iablokoff-Khnzorian, 1961: 86 [32]. Gender: masculine. Type species: Orthoraphes reichardti Iablokoff-Khnzorian, 1961: 87 [32]; by original designation. Eocene Baltic amber (38.0-33.9 Ma). Monotypic.

Literature. Iablokoff-Khnzorian (1961: 86) [32], Larsson (1978: 153) [54], Spahr (1981: 49) [37], Carpenter (1992: 305) [38], Alekseev (2013: 7) [39], Alekseev (2017: 409) [55]. 
- Elaterinae incertae sedis

Genus Crioraphes Iablokoff-Khnzorian, 1961

Crioraphes Iablokoff-Khnzorian, 1961: 93 [32]. Gender: masculine. Type species: Crioraphes rohdendorfi Iablokoff-Khnzorian, 1961: 94 [32]; by original designation. Eocene Baltic amber (38.0-33.9 Ma). Monotypic.

Literature. Iablokoff-Khnzorian (1961: 93) [32], Larsson (1978: 153) [54], Spahr (1981: 46) [37], Carpenter (1992: 304) [38], Douglas (2011: 17) [74], Alekseev (2013: 7) [39], Alekseev (2017: 409) [55], Douglas (2017: 4) [63].

Remark. This genus was originally placed in Cardiophorinae [32], however, Douglas [74] transferred it to Elaterinae incertae sedis based on the morphological phylogenetic analysis.

3.1.5. Subfamily Negastriinae Nakane \& Kishii, $1956^{*}$

Negastriinae Nakane \& Kishii, 1956: 203 [75]. Type genus: Negastrius Thomson, 1859.

Genus Ganestrius Dolin, 1976

Ganestrius Dolin, 1976: 69 [20]. Gender: masculine. Type species: Ganestrius stibicki Dolin, 1976: 71 [20]; by original designation. Jurassic of Kazakhstan (166.1-157.3 Ma). Two species.

Literature. Dolin (1976: 69) [20], Dolin (1980: 77) [13], Carpenter (1992: 304) [38], Korneev \& Cate (2005: 10) [46].

Genus Protoquasimus Dolin, 1976

Protoquasimus Dolin, 1976: 69 [20]. Gender: masculine. Type species: Protoquasimus brevicollis Dolin, 1976: 69 [20]; by original designation. Jurassic of Kazakhstan (166.1-157.3 Ma). Monotypic.

Literature. Dolin (1976: 69) [20], Dolin (1980: 76) [13], Carpenter (1992: 305) [38], Korneev \& Cate (2005: 10) [46].

3.1.6. Subfamily Omalisinae Lacordaire, $1857^{*}$

Homalisides Lacordaire, 1857: 303 [76]. Type genus: Omalisus Geoffroy, 1762.

Genus Jantarokrama Kirejtshuk \& Kovalev, 2015

Jantarokrama Kirejtshuk \& Kovalev, 2015: 1413 [77]. Gender: feminine. Type species: Jantarokrama utilis Kirejtshuk \& Kovalev, 2015: 1414 [77]; by original designation. Eocene Baltic amber (38.0-33.9 Ma). Monotypic.

Literature. Kirejtshuk \& Kovalev (2015: 1413) [77], Alekseev (2017: 410) [55].

3.1.7. Subfamily Pityobiinae Hyslop, $1917^{*}$

Pityobiinae Hyslop, 1917: 249 [78]. Type genus: Pityobius LeConte, 1853.

Genus Cretopityobius Otto, 2019

Cretopityobius Otto, 2019: 4 [27]. Gender: masculine. Type species: Cretopityobius pankowskiorum Otto, 2019: 4 [27]; by original designation. Cretaceous of Myanmar (Burmese amber) (99.6-93.5 Ma). Monotypic.

Literature. Otto (2019: 4) [27]. 
3.1.8. Subfamily Protagrypninae Dolin, 1973

Protagrypnini Dolin, 1973: 74 [18]. Type genus: Protagrypnus Dolin, 1973.

- $\quad$ Tribe Desmatini Dolin, 1975

Desmatini Dolin, 1975: 60 [19]. Type genus: Desmatus Dolin, 1975.

Genus Anoixis Chang, Kirejtshuk \& Ren, 2010

Anoixis Chang, Kirejtshuk \& Ren, 2010: 872 [25]. Gender: feminine. Type species: Anoixis complanus Chang, Kirejtshuk \& Ren, 2010: 873 [25]; by original designation. Cretaceous of China (125.5-122.5 Ma). Monotypic.

Literature. Chang et al. (2010: 872) [25], Dong \& Huang (2011: 1225) [79], Yu et al. (2019: 383) [80].

Genus Apoclion Chang, Kirejtshuk \& Ren, 2010

Apoclion Chang, Kirejtshuk \& Ren, 2010: 869 [25]. Gender: masculine. Type species: Apoclion clavatus Chang, Kirejtshuk \& Ren, 2010: 870 [25]; by original designation. Cretaceous of China (125.5-122.5 Ma). Three species.

Literature. Chang et al. (2010: 869) [25], Dong \& Huang (2011: 1225) [79], Yu et al. (2019: 383) [80].

Genus Desmatinus Chang, Kirejtshuk \& Ren, 2010

Desmatinus Chang, Kirejtshuk \& Ren, 2010: 868 [25]. Gender: masculine. Type species: Desmatinus cognatus Chang, Kirejtshuk \& Ren, 2010: 869 [25]; by original designation. Cretaceous of China (125.5-122.5 Ma). Monotypic.

Literature. Chang et al. (2010: 868) [25], Dong \& Huang (2011: 1225) [79], Yu et al. (2019: 383) [80].

Genus Desmatus Dolin, 1975

Desmatus Dolin, 1975: 60 [19]. Gender: masculine. Type species: Desmatus lapidarius Dolin, 1975 : 61 [19]; by original designation. Jurassic of Kazakhstan (166.1-157.3 Ma). Four species.

Literature. Dolin (1975: 60) [19], Dolin (1980: 64) [13], Carpenter (1992: 304) [38], Korneev \& Cate (2005: 10) [46], Chang et al. (2008: 60) [81], Chang et al. (2009: 8) [17], Chang et al. (2010: 869) [25].

Genus Paradesmatus Chang, Kirejtshuk \& Ren in Chang et al., 2009

Paradesmatus Chang, Kirejtshuk \& Ren in Chang et al., 2009: 8 [17]. Gender: masculine. Type species: Paradesmatus baie Chang, Kirejtshuk \& Ren, 2009: 8 [17]; by original designation. Cretaceous of China (125.5-122.5 Ma), Jurassic of China (166.1-157.3 Ma). Three species.

Literature. Chang et al. (2009: 8) [17], Chang et al. (2010: 867) [25], Kirejtshuk et al. (2010: 791) [82], Dong \& Huang (2011: 1225) [79], Yu et al. (2019: 382) [80].

Genus Plesiorhaphes Dolin, 1980

Plesiorhaphes Dolin, 1980: 65 [13]. Gender: masculine. Type species: Plesiorhaphes scaber Dolin, 1980: 66 [13]; by original designation. Jurassic of Kazakhstan (166.1-157.3 Ma). Monotypic.

Plesioraphes: Korneev \& Cate, 2005: 10 [46]; unavailable name, incorrect subsequent spelling not in prevailing usage [41].

Literature. Dolin (1980: 65) [13], Carpenter (1992: 305) [38], Korneev \& Cate (2005: 10) [46], Chang et al. (2008: 60) [81], Chang et al. (2010: 867) [25]. 
- $\quad$ Tribe Hypnomorphini Dolin, 1975

Hypnomorphini Dolin, 1975: 54 [19]. Type genus: Hypnomorphus Dolin, 1975.

Hipnomorphini: Ponomarenko et al., 2012: 482 [83]; unavailable name, incorrect subsequent spelling not in prevailing usage [41].

Genus Abrotus Dolin, 1980

Abrotus Dolin, 1980: 62 [13]. Gender: masculine. Type species: Abrotus sepultus Dolin, 1980: 63 [13]; by original designation. Jurassic of Kazakhstan (166.1-157.3 Ma). Two species.

Literature. Dolin (1980: 62) [13], Carpenter (1992: 304) [38], Korneev \& Cate (2005: 9) [46], Chang et al. (2008: 60) [81], Chang et al. (2011: 36) [84].

Remark. The non-type species of this genus was considered a member of Cerophytidae by Chang et al. [84], and the authors also called for a re-examination of the type species to test the systematic position of Abrotus.

Genus Adiagnostus Dolin, 1980

Adiagnostus Dolin, 1980: 44 [13]. Gender: masculine. Type species: Adiagnostus cardiophorinus Dolin, 1980: 45 [13]; by original designation. Jurassic of Kazakhstan (166.1-157.3 Ma). Three species.

Literature. Dolin (1980: 44) [13], Carpenter (1992: 304) [38], Korneev \& Cate (2005: 9) [46], Chang et al. (2008: 60) [81], Alekseev (2011: 424) [26].

Genus Codemus Dolin, 1980

Codemus Dolin, 1980: 35 [13]. Gender: masculine. Type species: Codemus synaptoides Dolin, 1980: 36 [13]; by original designation. Jurassic of Kazakhstan (166.1-157.3 Ma). 10 species.

Literature. Dolin (1980: 35) [13], Carpenter (1992: 304) [38], Korneev \& Cate (2005: 10) [46], Chang et al. (2008: 60) [81], Alekseev (2011: 424) [26].

Genus Elaterophanes Handlirsch, 1906

Elaterophanes Handlirsch, 1906: 436 [35]. Gender: masculine. Type species: Elater socius Giebel, 1856: 91 [85] (synonym of Elater vetustus Brodie, 1845: 101 [12]); by subsequent designation (Hyslop 1921: 644 [36]). Later designations by Whalley [14] (E. vetustus) and Carpenter [38] (E. socius) are invalid. Triassic of the United Kingdom (208.5-201.3 Ma), Jurassic of the United Kingdom (196.5-189.6 Ma). Three species.

Literature. Handlirsch (1906: 436) [35], Cockerell (1915: 478) [86], Hyslop (1921: 644) [36], Dolin (1973: 73) [18], Dolin (1975: 51) [19], Whalley (1985: 165) [14], Carpenter (1992: 304) [38].

Genus Graciolacon Dolin, 1980

Graciolacon Dolin, 1980: 61 [13]. Gender: masculine. Type species: Graciolacon aeternus Dolin, 1980: 62 [13]; by original designation. Jurassic of Kazakhstan (166.1-157.3 Ma). Monotypic.

Literature. Dolin (1980: 61) [13], Carpenter (1992: 304) [38], Korneev \& Cate (2005: 10) [46], Chang et al. (2008: 60) [81].

Genus Hypnomorphoides Dolin, 1980

Hypnomorphoides Dolin, 1980: 54 [13]. Gender: masculine. Type species: Hypnomorphoides catachtonius Dolin, 1980: 55 [13]; by original designation. Jurassic of Kazakhstan (166.1-157.3 Ma). Four species.

Literature. Dolin (1980: 54) [13], Carpenter (1992: 304) [38], Korneev \& Cate (2005: 10) [46], Chang et al. (2008: 60) [81]. 
Genus Hypnomorphus Dolin, 1975

Hypnomorphus Dolin, 1975: 54 [19]; erroneously published as gen. nov. also by Dolin (1980: 26) [13]. Gender: masculine. Type species: Hypnomorphus rohdendorfi Dolin, 1975: 56 [19]; by original designation. Jurassic of Kazakhstan (166.1-157.3 Ma). 14 species.

Hyponomorphus: Chang et al., 2008: 60 [81]; unavailable name, incorrect subsequent spelling not in prevailing usage [41].

Literature. Dolin (1975: 54) [19], Dolin (1980: 26) [13], Carpenter (1992: 304) [38], Korneev \& Cate (2005: 10) [46], Chang et al. (2008: 60) [81], Dong et al. (2011: 482) [87].

Genus Idiomorphus Dolin, 1980

Idiomorphus Dolin, 1980: 60 [13]. Gender: masculine. Type species: Idiomorphus singularis Dolin, 1980: 60 [13]; by original designation. Jurassic of Kazakhstan (166.1-157.3 Ma). Two species.

Literature. Dolin (1980: 60) [13], Carpenter (1992: 304) [38], Korneev \& Cate (2005: 10) [46], Chang et al. (2008: 60) [81].

Genus Lapidiconides Dolin, 1980

Lapidiconides Dolin, 1980: 43 [13]. Gender: masculine. Type species: Lapidiconides excellens Dolin, 1980: 43 [13]; by original designation. Jurassic of Kazakhstan (166.1-157.3 Ma). Three species.

Lapidioconides: Korneev \& Cate: 10 [46]; unavailable name, incorrect subsequent spelling not in prevailing usage [41].

Literature. Dolin (1980: 43) [13], Carpenter (1992: 304) [38], Korneev \& Cate (2005: 10) [46], Chang et al. (2008: 60) [81].

Genus Lapidostenus Dolin, 1980

Lapidostenus Dolin, 1980: 30 [13]. Gender: masculine. Type species: Lapidostenus infossus Dolin, 1980: 31 [13]; by original designation. Jurassic of Kazakhstan (166.1-157.3 Ma). Five species.

Literature. Dolin (1980: 30) [13], Carpenter (1992: 304) [38], Korneev \& Cate (2005: 10) [46], Chang et al. (2008: 60) [81].

Genus Lithoptychus Dolin, 1980

Lithoptychus Dolin, 1980: 57 [13]. Gender: masculine. Type species: Lithoptychus handlirschi Dolin, 1980: 57 [13]; by original designation. Jurassic of Kazakhstan (166.1-157.3 Ma). Four species.

Literature. Dolin (1980: 57) [13], Carpenter (1992: 305) [38], Korneev \& Cate (2005: 10) [46], Chang et al. (2008: 60) [81].

Genus Lithosomus Dolin, 1980

Lithosomus Dolin, 1980: 46 [13]. Gender: masculine. Type species: Lithosomus erosus Dolin, 1980: 47 [13]; by original designation. Jurassic of Kazakhstan (166.1-157.3 Ma). Two species.

Literature. Dolin (1980: 46) [13], Carpenter (1992: 305) [38], Korneev \& Cate (2005: 10) [46], Chang et al. (2008: 60) [81].

Genus Necrocoelus Dolin, 1980

Necrocoelus Dolin, 1980: 59 [13]. Gender: masculine. Type species: Necrocoelus aselloides Dolin, 1980: 59 [13]; by original designation. Jurassic of Kazakhstan (166.1-157.3 Ma). Monotypic.

Necrocelus: Carpenter, 1992: 305 [38]; unavailable name, incorrect subsequent spelling not in prevailing usage [41].

Literature. Dolin (1980: 59) [13], Carpenter (1992: 305) [38], Korneev \& Cate (2005: 10) [46], Chang et al. (2008: 60) [81]. 
Genus Negastrioides Dolin, 1980

Negastrioides Dolin, 1980: 52 [13]. Gender: masculine. Type species: Negastrioides tenuis Dolin, 1980: 52 [13]; by original designation. Jurassic of Kazakhstan (166.1-157.3 Ma). Four species.

Negastroides: Korneev \& Cate, 2005: 15 [46]; unavailable name, incorrect subsequent spelling not in prevailing usage [41].

Literature. Dolin (1980: 52) [13], Carpenter (1992: 305) [38], Korneev \& Cate (2005: 10) [46], Chang et al. (2008: 60) [81].

Genus Parahypnomorphus Dolin, 1980

Parahypnomorphus Dolin, 1980: 33 [13]. Gender: masculine. Type species: Parahypnomorphus jurassicus Dolin, 1980: 33 [13]; by original designation. Jurassic of Kazakhstan (166.1-157.3 Ma). Three species.

Literature. Dolin (1980: 33) [13], Carpenter (1992: 305) [38], Korneev \& Cate (2005: 10) [46], Chang et al. (2008: 60) [81].

Genus Platyelater Dolin, 1980

Platyelater Dolin, 1980: 40 [13]. Gender: masculine. Type species: Platyelater reflexicollis Dolin, 1980: 41 [13]; by original designation. Jurassic of Kazakhstan (166.1-157.3 Ma). Four species.

Platyelata: Carpenter, 1992: 305 [38]; unavailable name, incorrect subsequent spelling not in prevailing usage [41].

Literature. Dolin (1980: 40) [13], Carpenter (1992: 305) [38], Korneev \& Cate (2005: 10) [46], Chang et al. (2008: 60) [81], Dong et al. (2011: 482) [87].

- $\quad$ Tribe Pollostelaterini Alekseev, 2011

Pollostelaterini Alekseev, 2011: 424 [26]. Type genus: Pollostelater Alekseev, 2011.

Pollostelasterini: Sohn et al., 2019: 9 [28]; unavailable name, incorrect subsequent spelling not in prevailing usage [41].

Genus Pollostelater Alekseev, 2011

Pollostelater Alekseev, 2011: 424 [26]. Gender: masculine. Type species: Pollostelater baissensis Alekseev, 2011: 424 [26]; by original designation. Cretaceous of Russian Federation (125.0-113.0 Ma). Monotypic.

Literature: Alekseev (2011: 424) [26].

- $\quad$ Tribe Protagrypnini Dolin, 1973

Protagrypnini Dolin, 1973: 74 [18]. Type genus: Protagrypnus Dolin, 1973.

Genus Acheonus Dolin, 1980

Acheonus Dolin, 1980: 20 [13]. Gender: masculine. Type species: Acheonus abbreviatus Dolin, 1980: 21 [13]; by original designation. Jurassic of Kazakhstan (166.1-157.3 Ma). Three species.

Archeonus: Carpenter, 1992: 304 [38]; unavailable name, incorrect subsequent spelling not in prevailing usage [41].

Literature. Dolin (1980: 20) [13], Carpenter (1992: 304) [38], Korneev \& Cate (2005: 9) [46], Chang et al. (2008: 60) [81], Alekseev (2011: 424) [26], Dong et al. (2011: 482) [87]. 
Genus Archaeolus Lin, 1986

Archaeolus Lin, 1986: 78 [21]. Gender: masculine. Type species: Archaeolus funestus Lin, 1986: 78 [21]; by original designation. Jurassic of China (170.3-168.3 Ma). Monotypic.

Literature: Lin (1986: 78) [21], Zhang (1997: 71) [88], Dolin \& Nel (2002: 345) [89], Dong \& Huang (2009: 102) [22], Dong \& Huang (2011: 1225) [79], Dong et al. (2011: 481) [87], Ponomarenko et al. (2012: 479) [83], Yu et al. (2019: 409) [80].

Genus Clavelater Dong \& Huang, 2011

Clavelater Dong \& Huang, 2011: 1225 [79]. Gender: masculine. Type species: Clavelater ningchengensis Dong \& Huang, 2011: 1226 [79]; by monotypy. Jurassic of China (166.1-157.3 Ma). Monotypic.

Literature: Dong \& Huang (2011: 1225) [79], Yu et al. (2019: 383) [80].

Genus Koreagrypnus Sohn \& Nam in Sohn et al., 2019

Koreagrypnus Sohn \& Nam in Sohn et al., 2019: 6 [28]. Gender: masculine. Type species: Koreagrypnus jinju Sohn \& Nam in Sohn et al., 2019: 6 [28]; by original designation. Cretaceous of South Korea (113.0-100.5 Ma). Monotypic.

Literature. Sohn et al. (2019: 6) [28].

Genus Lithocoelus Dolin, 1975

Lithocoelus Dolin, 1975: 53 [19]. Gender: masculine. Type species: Lithocoelus detrusus Dolin, 1975: 53 [19]; by original designation. Jurassic of Kazakhstan (166.1-157.3 Ma). Two species.

Literature. Dolin (1975: 53) [19], Dolin (1980: 20) [13], Carpenter (1992: 305) [38], Korneev \& Cate (2005: 10) [46], Chang et al. (2008: 60) [81].

Genus Lithomerus Dolin, 1980

Lithomerus Dolin, 1980: 23 [13]. Gender: masculine. Type species: Lithomerus cockerelli Dolin, 1980: 23 [13]; by original designation. Jurassic of Australia (182.7-174.1 Ma), Jurassic of Kazakhstan (166.1-157.3 Ma), Cretaceous of China (125.5-122.5 Ma). Six species.

Literature. Dolin (1980: 23) [13], Carpenter (1992: 305) [38], Dolin \& Nel (2002: 341) [89], Korneev \& Cate (2005: 10) [46], Chang et al. (2008: 55) [81], Dong \& Huang (2009: 103) [22], Kirejtshuk et al. (2010: 792) [82], Martin (2010: 932) [90], Dong \& Huang (2011: 1225) [79], Ponomarenko et al. (2012: 479) [83], Sohn et al. (2019: 3) [28], Yu et al. (2019: 382) [80].

Genus Megalithomerus Sohn \& Nam in Sohn et al., 2019

Megalithomerus Sohn \& Nam in Sohn et al., 2019: 3 [28]. Gender: masculine. Type species: Megalithomerus magohalmii Sohn \& Nam in Sohn et al., 2019: 3 [28]; by original designation. Cretaceous of South Korea (113.0-100.5 Ma). Monotypic.

Literature. Sohn et al. (2019: 3) [28].

Genus Micragrypnites Dolin, 1973

Micragrypnites Dolin, 1973: 76 [18]. Gender: masculine. Type species: Micragrypnites issykiensis Dolin, 1973: 77 [18]; by original designation. Jurassic of Kyrgyzstan (201.3-190.8 Ma). Monotypic.

Literature. Dolin (1973: 76) [18], Dolin (1975: 51) [19], Carpenter (1992: 305) [38], Korneev \& Cate (2005: 10) [46], Chang et al. (2008: 60) [81], Dong \& Huang (2009: 103) [22], Dong et al. (2011: 482) [87]. 
Genus Paragrypnites Dolin, 1980

Paragrypnites Dolin, 1980: 22 [13]. Gender: masculine. Type species: Paragrypnites jagemanni Dolin, 1980: 22 [13]; by original designation. Jurassic of Kazakhstan (166.1-157.3 Ma). Monotypic.

Paragrypnus: Chang et al., 2008: 60 [81]; unavailable name, incorrect subsequent spelling not in prevailing usage [41].

Literature. Dolin (1980: 22) [13], Carpenter (1992: 305) [38], Korneev \& Cate (2005: 10) [46], Chang et al. (2008: 60) [81], Ponomarenko et al. (2012: 479) [83].

Genus Paraprotagrypnus Chang, Zhao \& Ren, 2009

Paraprotagrypnus Chang, Zhao \& Ren, 2009: 1433 [91]. Gender: masculine. Type species: Paraprotagrypnus superbus Chang, Zhao \& Ren, 2009: 1434 [91]; by original designation. Jurassic of China (166.1-157.3 Ma). Monotypic.

Literature. Chang et al. (2009: 1433) [91], Kirejtshuk et al. (2010: 791) [82], Dong \& Huang (2011: 1225) [79], Sohn et al. (2019: 3) [28], Yu et al. (2019: 382) [80].

Genus Protagrypnus Dolin, 1973

Protagrypnus Dolin, 1973: 75 [18]. Gender: masculine. Type species: Protagrypnus exoletus Dolin, 1973: 75 [18]; by original designation. Jurassic of Kyrgyzstan (201.3-190.8 Ma), Jurassic of China (166.1-157.3 Ma). Two species.

Protagrypnites: Chang et al., 2008: 60 [81]; unavailable name, incorrect subsequent spelling not in prevailing usage [41].

Literature. Dolin (1973: 75) [18], Dolin (1975: 51) [19], Carpenter (1992: 305) [38], Korneev \& Cate (2005: 10) [46], Chang et al. (2008: 60) [81], Chang et al. (2009: 10) [17], Chang et al. (2009: 1434) [91], Dong \& Huang (2009: 103) [22], Kirejtshuk et al. (2010: 791) [82], Dong \& Huang (2011: 1225) [79], Sohn et al. (2019: 3) [28], Yu et al. (2019: 381) [80].

Genus Sinolithomerus Dong \& Huang, 2009

Sinolithomerus Dong \& Huang, 2009: 103 [22]. Gender: masculine. Type species: Sinolithomerus dolini Dong \& Huang, 2009: 104 [22]; by original designation. Jurassic of China (166.1-157.3 Ma). Monotypic.

Literature. Dong \& Huang (2009: 103) [22], Dong \& Huang (2011: 1225) [79], Yu et al. (2019: 383) [80].

- Protagrypninae incertae sedis

Genus Paralithomerus Chang, Zhang \& Ren, 2008

Paralithomerus Chang, Zhang \& Ren, 2008: 55 [81]. Gender: masculine. Type species: Paralithomerus exquisitus Chang, Zhang \& Ren, 2008: 55 [81]; by original designation. Cretaceous of China (125.5-122.5 Ma). Two species.

Literature. Chang et al. (2008: 55) [81], Dong \& Huang (2009: 103) [22], Kirejtshuk et al. (2010: 792) [82], Dong \& Huang (2011: 1225) [79], Sohn et al. (2019: 3) [28], Yu et al. (2019: 382) [80].

Remark. This genus was originally described in Elateridae without any subfamilial assignment due to the presence of only one of two main diagnostic characters of Protagrypninae [81]. However, subsequent authors placed Paralithomerus into Protagrypninae [28,82]. 


\subsubsection{Elateridae incertae sedis}

Genus Adocetus Scudder, 1900

Adocetus Scudder, 1900: 97 [92]. Gender: masculine. Type species: Adocetus buprestoides Scudder, 1900: 97 [92]; by monotypy. Eocene of the United States (55.8-50.3 Ma). Monotypic.

Literature. Scudder (1900: 97) [92], Handlirsch (1907: 747) [93], Carpenter (1992: 304) [38].

Remark. Both Scudder [92] and Carpenter [38] hypothesized the close relationship between Adocetus and Scaptolenus LeConte, 1853 (Elaterinae: Cebrionini) but the shape of prothorax in Adocetus is completely different.

Genus Artinama Lin, 1986

Artinama Lin, 1986: 72 [21]. Gender: feminine. Type species: Artinama qinghuoensis Lin, 1986: 73 [21]; by original designation. Jurassic of China (199.3-190.8 Ma). Monotypic.

Literature. Lin (1986: 72) [21], Dong \& Huang (2011: 1225) [79], Dong et al. (2011: 482) [87], Ponomarenko et al. (2012: 477) [83], Yu et al. (2019: 410) [80].

Remark. It was originally placed in Acanthocnemidae, although with a question mark [21]. Dong et al. [87] placed it in Elateridae, again with a question mark. Ponomarenko et al. [83] hypothesized that Artinama belongs either to Praelateridae (currently synonymized with Cerophytidae by Yu et al. [94]) or Elateridae: Protagrypninae.

Genus Bilineariselater Chang \& Ren, 2008

Bilineariselater Chang \& Ren, 2008: 237 [24]. Gender: masculine. Type species: Bilineariselater foveatus Chang \& Ren, 2008: 237 [24]; by original designation. Cretaceous of China (125.5-122.5 Ma). Monotypic.

Literature. Chang \& Ren (2008: 237) [24], Kirejtshuk et al. (2010: 792) [82], Dong \& Huang (2011: 1225) [79], Yu et al. (2019: 382) [80].

Remark. Chang \& Ren [24] did not assign this genus to any subfamily due to the lack of diagnostic characters, although they hypothesized its relationship to either Agrypninae or Elaterinae. Kirejtshuk et al. [82] listed this genus under Protagrypninae but without any justification or clarification. Therefore, we here follow the original placement by Chang \& Ren [24].

Genus Cretoelaterium Alekseev, 2008

Cretoelaterium Alekseev, 2008: 56 [95]. Gender: neuter. Type species: Cretoelaterium kazanovense Alekseev, 2008: 57 [95]; by original designation. Cretaceous of Russian Federation (129.4-125.0 Ma). Monotypic.

Literature. Alekseev (2008: 56) [95].

Remark. Alekseev [95] classified this genus in Elateridae without any subfamilial assignment.

Genus Cryptocoelus Dolin \& Nel, 2002

Cryptocoelus Dolin \& Nel, 2002: 342 [89]. Gender: masculine. Type species: Cryptocoelus buffoni Dolin \& Nel, 2002: 342 [89]; by original designation (not C. major Dolin \& Nel, 2002: 343 as erroneously stated by Korneev \& Cate [46] and Chang et al. [56]). Cretaceous of China (125.5-122.5 Ma), Cretaceous of Russian Federation (125.0-113.0 Ma). Eight species.

Cryptocoleous: Chang et al., 2007: 1245 [56]; unavailable name, incorrect subsequent spelling not in prevailing usage [41].

Crytocoelus: Yu et al., 2019: 381 [80]; unavailable name, incorrect subsequent spelling not in prevailing usage [41].

Literature. Dolin \& Nel (2002: 342) [89], Korneev \& Cate (2005: 10) [46], Chang et al. (2007: 1245) [56], Chang \& Ren (2008: 237) [24], Chang et al. (2009: 8) [17], Dong \& Huang (2009: 102) [22], 
Chang et al. (2010: 873) [25], Kirejtshuk et al. (2010: 792) [82], Alekseev (2011: 424) [26], Dong \& Huang (2011: 1225) [79], Yu et al. (2019: 381) [80].

Remark. This genus was originally classified in the agrypnine tribe Cryptocardiini [89], and its placement in Agrypninae was followed by Kirejtshuk et al. [82]. However, Chang et al. [56] revised the morphology of Cryptocoelus and classified it in Elateridae incertae sedis, which was followed by Alekseev [26].

Genus Curtelater Chang \& Ren, 2008

Curtelater Chang \& Ren, 2008: 238 [24]. Gender: masculine. Type species: Curtelater wui Chang \& Ren, 2008: 239 [24]; by original designation. Cretaceous of China (125.5-122.5 Ma). Monotypic.

Literature. Chang \& Ren (2008: 238) [24], Kirejtshuk et al. (2010: 792) [82], Dong \& Huang (2011: 1225) [79], Yu et al. (2019: 382) [80].

Remark. Chang \& Ren [24] did not assign this genus to any subfamily because of the lack of diagnostic characters, although they hypothesized its relationship to either Agrypninae or Elaterinae. Kirejtshuk et al. [82] listed this genus under Protagrypninae but without any justification or clarification. Therefore, we here follow the original placement by Chang \& Ren [24].

Genus Elateridium Tillyard, 1918

Elaterites Tillyard, 1916: 41 [96]. Preoccupied by Elaterites Heer, 1847: 141 [97] [Coleoptera: Elateridae]. Elateridium Tillyard, 1918: 751 [98]. Replacement name for Elaterites Tillyard, 1916. Gender: neuter. Type species: Elaterites wianamattensis Tillyard, 1916: 41 [96]; by original designation. Triassic of Australia (247.2-208.5 Ma). Three species.

Elaterium: Ponomarenko, 2011: 421 [99]; unavailable name, incorrect subsequent spelling of Elateridium Tillyard, 1918, not in prevailing usage [41]. Preoccupied by Elaterium Westwood, 1854.

Literature. Tillyard (1916: 41) [96], Tillyard (1918: 751) [98], Dunstan (1923: 44) [100], Handlirsch (1938: 13) [101], Carpenter (1992: 304) [38], Jell (2004: 76) [102], Martins-Neto et al. (2006: 602) [103], Ponomarenko (2011: 421) [99].

Remark. This genus contains species described based on a single elytron each $[96,100]$, and its systematic placement remains unclear.

Genus Elaterites Heer, 1847

Elaterites Heer, 1847: 141 [97]. Gender: masculine. Type species: Elaterites lavateri Heer, 1847: 141 [97]; by subsequent designation (Handlirsch 1907: 747 [93]). Paleocene of Argentina (66.0-56.0 Ma), Eocene of the United Kingdom (56.0-41.3 Ma), Eocene of Germany (47.8-41.3 Ma), Eocene of Czech Republic (37.2-33.9 Ma), Oligocene of Switzerland (28.4-23.0 Ma), Miocene of Germany (12.7-11.6 Ma). 12 species.

Literature. Heer (1847: 141) [97], Giebel (1856: 94) [85], Deichmüller (1881: 308) [104], Scudder (1885: 797) [105], Scudder (1886: 77) [106], Scudder (1891: 205) [34], Scudder (1900: 98) [92], Handlirsch (1906: 450) [35], Handlirsch (1907: 747) [93], Cockerell (1920: 456) [107], Dunstan (1923: 44) [100], Cockerell (1926: 320) [108], Haupt (1956: 48) [58], Martins-Neto et al. (2006: 602) [103].

Remark. Heer [97] erected this genus to accommodate the species which he was unable to place to any existing genus at that time. This genus is a waste basket for fossils with no clear characters (mostly represented by isolated elytra) and may contain representatives of many genera [36,97,107].

Genus Elaterium Westwood, 1854

Elaterium Westwood, 1854: 387/393 [109]. Gender: masculine. Type species: Elaterium pronaeus Westwood, 1854: 387/393 [109]; by subsequent monotypy in Handlirsch (1906: 553) [35], not by subsequent designation [36]. Triassic of Australia (228.0-208.5 Ma), Cretaceous of the United Kingdom (145.0-140.2 Ma). Two species. 
Literature. Westwood (1854: 387/393) [109], Giebel (1856: 92) [85], Scudder (1885: 797) [105], Scudder (1886: 77) [106], Scudder (1891: 518) [34], Handlirsch (1906: 553) [35], Handlirsch (1907: 748) [93], Hyslop (1921: 644) [36], Dunstan (1923: 46) [100], Cockerell (1920: 456) [107], Handlirsch (1938: 14) [101], Jell (2004: 76) [102], Coram \& Jepson (2012: 60) [110], Kirejtshuk (2020: 18) [111].

Remark. This genus has never been classified to any existing elaterid subfamily and its position remains unclear.

Genus Gripecolous Lin, 1986

Gripecolous Lin, 1986: 80 [21]. Gender: masculine. Type species: Gripecolous enallus Lin, 1986: 80 [21]; by original designation. Jurassic of China (170.3-168.3 Ma). Monotypic.

Literature. Lin (1986: 80) [21], Dong \& Huang (2011: 1225) [79], Dong et al. (2011: 482) [87], Ponomarenko et al. (2012: 482) [83], Yu et al. (2019: 410) [80].

Remark. This genus was originally described in Silphidae [21]. Dong et al. [87] listed it in Protagrypninae, Ponomarenko et al. [83] hypothesized that Gripecolous belongs either to Praelateridae or Elateridae: Protagrypninae, and Yu et al. [80] listed it in Elateridae without further details.

Genus Ludiophanes Wickham, 1916

Ludiophanes Wickham, 1916: 522 [29]. Gender: masculine. Type species: Ludiophanes haydeni Wickham, 1916: 522 [29]; by original designation. Eocene of the United States (38.0-33.9 Ma). Monotypic.

Literature. Wickham (1916: 522) [29], Hyslop (1921: 654) [36], Carpenter (1992: 305) [38].

Remark. This genus has never been classified to any existing elaterid subfamily although Wickham [29] and Carpenter [38] hypothesized its affinities to Ludius Latreille, 1834 (currently a synonym of Elater Linnaeus, 1758) and Megapenthes Kiesenwetter, 1858, respectively, which both are currently classified in Elaterinae.

Genus Mercata Lin, 1986

Mercata Lin, 1986: 79 [21]. Gender: feminine. Type species: Mercata festira Lin, 1986: 79 [21]; by original designation. Jurassic of China (170.3-168.3 Ma). Monotypic.

Literature. Lin (1986: 79) [21], Chang et al. (2011: 33) [84], Chang et al. (2011: 701) [112], Dong \& Huang (2011: 1225) [79], Dong et al. (2011: 486) [87], Ponomarenko et al. (2012: 480) [83], Oberprieler et al. (2016: 177) [113], Kundrata \& Jäch (2017: 371) [114], Yu et al. (2019: 410) [80].

Remarks. This genus was originally classified in Silphidae [21] but later transferred to Cerophytidae by Chang et al. [84]. The latter authors provided no evidence for the placement of Mercata in Cerophytidae, and in other publication they wrote that it is questionable [112]. Mercata was not included in the most recent revision of the fossil Cerophytidae [94]. Ponomarenko et al. [83] classified Mercata in Elateridae: Protagrypninae without any tribal assignment, and Yu et al. [80] placed it in Elateridae without further details.

Genus Ovivagina Zhang, 1997

Ovivagina Zhang, 1997: 71 [88]. Gender: feminine. Type species: Ovivagina longa Zhang, 1997: 72 [88]; by original designation. Jurassic of China (201.3-190.8 Ma). Monotypic.

Literature. Zhang (1997: 71) [88], Dolin \& Nel (2002: 345) [89], Chang et al. (2009: 8) [17], Dong \& Huang (2009: 102) [22], Yan \& Zhang (2010: 451) [115], Dong \& Huang (2011: 1225) [79], Yan et al. (2013: 43) [116], Yu et al. (2019: 410) [80].

Remark. Zhang [88] described five species of this genus, but all of them except the type species, $O$. longa, were transferred to the genus Artematopodites Ponomarenko, 1990 [115], which has an uncertain position within Coleoptera [117-119]. The placement of O. longa in Elateridae is also dubious [17,79,115]. 
Genus Protocardiophorus Dolin, 1976

Protocardiophorus Dolin, 1976: 71 [20]. Gender: masculine. Type species: Protocardiophorus ancestralis Dolin, 1976: 73 [20]; by original designation. Jurassic of Kazakhstan (166.1-157.3 Ma). Two species.

Literature. Dolin (1976: 71) [20], Dolin (1980: 78) [13], Carpenter (1992: 305) [38], Korneev \& Cate (2005: 10) [46], Douglas (2011: 19) [74], Douglas (2017: 4) [63].

Remark. This genus was originally placed in Cardiophorinae [13,20], however, Douglas [74] did not confirm this placement based on the morphological phylogenetic analysis.

Genus Pseudocardiophorites Dolin, 1976

Pseudocardiophorites Dolin, 1976: 73 [20]. Gender: masculine. Type species: Pseudocardiophorites fragilis Dolin, 1976: 73 [20]; by original designation. Jurassic of Kazakhstan (166.1-157.3 Ma). Five species.

Literature. Dolin (1976: 73) [20], Dolin (1980: 79) [13], Carpenter (1992: 305) [38], Korneev \& Cate (2005: 10) [46], Douglas (2011: 19) [74], Douglas (2017: 4) [63].

Remark. This genus was originally placed in Cardiophorinae [13,20], however, Douglas [74] did not confirm this placement based on the morphological phylogenetic analysis.

Genus Silicernius Heyden, 1859

Silicernius Heyden, 1859: 6 [120]. Gender: masculine. Type species: Silicernius spectabilis Heyden, 1859: 6 [120]; by monotypy. Oligocene of Germany (28.4-23.0 Ma). Monotypic.

Literature. Heyden (1859: 6) [120], Scudder (1885: 797) [105], Scudder (1886: 77) [106], Scudder (1891: 580) [34], Handlirsch (1907: 747) [93], Hyslop (1921: 669) [36].

Remark. This genus has never been classified to any existing elaterid subfamily and its position remains unclear.

Genus Sinoelaterium Ping, 1928

Sinoelaterium Ping, 1928: 22 [23]. Gender: neuter. Type species: Sinoelaterium melanocolor Ping, 1928: 23 [23]; by original designation. Cretaceous of China (125.5-122.5 Ma). Monotypic.

Literature. Ping (1928: 22) [23], Handlirsch (1938: 167) [101], Carpenter (1992: 305) [38], Dolin \& Nel (2002: 345) [89], Chang et al. (2009: 8) [17], Dong \& Huang (2009: 102) [22], Dong \& Huang (2011: 1225) [79], Yu et al. (2019: 409) [80].

Remark. Ping [23] described Sinoelaterium in Elateridae. Crowson [121] and Hörnschemeyer [122] hypothesized that it might belong to Artematopodidae. However, most authors placed Sinoelaterium into Elateridae, although with some reservations [38,80,101].

Genus Tetraraphes Iablokoff-Khnzorian, 1961

Tetraraphes Iablokoff-Khnzorian, 1961: 95 [32]. Gender: masculine. Type species: Tetraraphes ebersini Iablokoff-Khnzorian, 1961: 96 [32]; by original designation. Eocene Baltic amber (38.0-33.9 Ma). Monotypic.

Literature. Iablokoff-Khnzorian (1961: 95) [32], Larsson (1978: 153) [54], Zherikhin (1980: 60) [123], Spahr (1981: 49) [37], Carpenter (1992: 305) [38], Chang et al. (2010: 867) [25], Alekseev (2013: 7) [39], Alekseev (2017: 409) [55].

Remark. This genus was originally described in Elateridae without any subfamilial assignment, and this was followed by subsequent authors. Chang et al. [25] discussed the morphological characters of Tetraraphes and its similarity in the shape of metacoxal plates to Desmatini but also listed characters in which these taxa differ. 
Genus Turonelater Alekseev, 2011

Turonelater Alekseev, 2011: 430 [26]. Gender: masculine. Type species: Turonelater giganteus Alekseev, 2011: 430 [26]; by original designation. Cretaceous of Kazakhstan (93.9-89.8 Ma). Monotypic.

Literature. Alekseev (2011: 430) [26].

Remark: This genus was classified as Elateridae incertae sedis by Alekseev [26].

\subsection{Fossil Genera Excluded Here from Elateridae}

Genus Babuskaya Martins-Neto \& Gallego, 2009

Babuskaya Martins-Neto \& Gallego, 2009: 368 [124]. Gender: feminine. Type species: Babuskaya elaterata Martins-Neto \& Gallego, 2009: 368 [124]; by original designation. Triassic of Argentina (237.0-228.0 Ma). Monotypic.

Literature. Martins-Neto \& Gallego (2009: 368) [124], Martins-Neto et al. (2011: 3) [125], Lara et al. (2012: 6) [126].

Remark. Martins-Neto \& Gallego [124] placed this genus in Elateridae with a question mark. Since the elytron, based on which this genus was described, shows none of the diagnostic characters of Elateridae, we transfer Babuskaya Martins-Neto \& Gallego, 2009 to Coleoptera incertae sedis.

Genus Cardiosyne Martins-Neto \& Gallego in Martins-Neto et al., 2006

Cardiosyne Martins-Neto \& Gallego in Martins-Neto et al., 2006: 602 [103]. Gender: feminine. Type species: Cardiosyne obesa Martins-Neto \& Gallego in Martins-Neto et al., 2006: 602 [103]; by original designation. Triassic of Argentina (237.0-228.0 Ma). Two species.

Literature. Martins-Neto et al. (2006: 602) [103], Martins-Neto et al. (2011: 3) [125], Lara et al. (2012: 6) [126].

Remark. Martins-Neto et al. [103] classified this genus in Elateridae only tentatively and hypothesized that it might in fact belong to a yet undescribed beetle family. Since the elytron, based on which this genus was described, shows none of the diagnostic characters of Elateridae, we transfer Cardiosyne Martins-Neto \& Gallego, 2006 to Coleoptera incertae sedis.

Genus Fengningia Hong, 1984

Fengningia Hong, 1984: 167 [127]. Gender: feminine. Type species: Fengningia punctata Hong, 1984: 167 [127]; by original designation. Cretaceous of China (125.0-113.0 Ma). Monotypic.

Literature. Hong (1984: 167) [127], Dong \& Huang (2011: 1225) [79], Yu et al. (2019: 409) [80].

Remark. The habitus line-drawing of the holotype by Hong [127] shows none of the diagnostic characters of Elateridae, which was already discussed by Dong \& Huang [79]. Therefore, we transfer Fengningia Hong, 1984 to Coleoptera incertae sedis.

Genus Gemelina Martins-Neto \& Gallego in Martins-Neto et al., 2006

Gemelina Martins-Neto \& Gallego in Martins-Neto et al., 2006: 602 [103]. Gender: feminine. Type species: Gemelina triangularis Martins-Neto \& Gallego in Martins-Neto et al., 2006: 602 [103]; by original designation. Triassic of Argentina (237.0-228.0 Ma). Monotypic.

Literature. Martins-Neto et al. (2006: 602) [103], Martins-Neto et al. (2011: 3) [125], Lara et al. (2012: 6) [126].

Remark. Martins-Neto et al. [103] placed this genus in Elateridae with a question mark. Since the elytron, based on which this genus was described, shows none of the diagnostic characters of Elateridae, we transfer Gemelina Martins-Neto \& Gallego, 2006 to Coleoptera incertae sedis. 


\section{Discussion}

Elateridae are among the most common beetle families in the fossil record $[16,54,128]$. However, their real palaeodiversity remains understudied [16], most probably because of their rather uniform and generally problematic external morphology which often causes problems even in identification and classification of recent lineages [1,3-11]. Consequently, the systematics and classification of fossil elaterids has been in a constant state of flux. Many genera that were listed as belonging to Elateridae by various authors in the past, have been currently classified in different beetle families, or they bear no diagnostic characters which would allow researchers to place them properly, and so they stay as incertae sedis.

Handlirsch $[35,93]$ listed in Elateridae many doubtful genera, usually described based on a single elytron, which can belong to any beetle family. This causes problems not only in Elateridae; for example, Nabozhenko [129] pointed out this issue and did not include Handlirsch's taxa in his catalogue of fossil Tenebrionidae. Although the systematic position of many genera listed by Handlirsch $[35,93]$ were not clear and some placements were questioned even by Handlirsch himself, Hyslop [36] listed many of them in his catalogue of the generic names in Elateridae, and added some more. The genera Apistotes Handlirsch, 1906, Biadelater Handlirsch, 1906, Parabuprestium Handlirsch, 1906, Micrelaterium Handlirsch, 1906 and Paragrilium Handlirsch, 1906 from the Cretaceous of the United Kingdom, Anepismus Handlirsch, 1906, Mimelater Handlirsch, 1906 and Stenelytron Handlirsch, 1906 from the Triassic of the United Kingdom, Dysarestus Handlirsch, 1906, Glaphyropterites Handlirsch, 1906 and Megacentrus Heer, 1852 from the Jurassic of Switzerland, Enamma Handlirsch, 1906 from the Jurassic of Germany, Pseudoelateropsis Handlirsch, 1906 (the replacement name for Elateropsis Roemer, 1876) from the Triassic of Germany, Plastelater Handlirsch, 1906 from the Triassic of the United Kingdom and Jurassic of Russian Federation, and Keleusticus Handlirsch, 1906 from the Jurassic of Germany, Mongolia and Russian Federation, are currently classified in Coleoptera incertae sedis [38]. Anchylocheira Giebel, 1856 was in fact a misspelling of Ancylochira Eschscholtz, 1829, which is currently in Buprestidae, and Hydriphilites Geinitz, 1884 was a misspelling of Hydrophilites Heer, 1865, which is classified in Coleoptera incertae sedis [38]. Glaphyroptera Heer, 1852 from the Jurassic of Switzerland was moved to its own family Glaphyropteridae [130] and later transferred to Buprestidae [44,131]. Pseudothyrea Handlirsch, 1906 from the Jurassic of Germany was also placed in Buprestidae [131,132]. Glaphyropterodes Handlirsch, 1906 from the Jurassic of Switzerland and Russian Federation was classified in Coleoptera incertae sedis [38,133] and currently it has been placed in the extinct elateriform family Lasiosynidae [118]. Malmelater Handlirsch, 1906 from the Jurassic of Germany, which was hypothesized by older authors to be the first fossil reliably placed in Elateridae [29,35], was transferred to Phoroschizidae (= Schizophoridae) [38,134]. The monotypic Mecynocanthus Hope, 1837 from the Holocene tropical fossil resin was synonymized with the extant genus Agrypnus Eschscholtz, 1829 [135].

Besides the problematic genera catalogued by Hyslop [36], there were a few other fossil genera classified in Elateridae which are currently considered not belonging to this family. Martynov [136] described genus Tersus from the Jurassic of Kazakhstan, in Elateridae. Although Crowson [121] suggested this genus might belong to Ptilodactylidae, it has been currently classified in the archostematan family Phoroschizidae (= Schizophoridae) [38,137,138]. The genus Idiomerus Dolin, 1980 from the Jurassic of Kazakhstan was originally described in Elateridae [13,38] but later synonymized with the genus Necromera Martynov, 1926 (Cerophytidae) by Chang et al. [84], which was followed by subsequent authors [80,94,113,114]. The monotypic Elaterina Gardiner, 1961 from the Jurassic of the United Kingdom was originally placed in Elateridae, but Whalley [14] questioned that placement because the holotype elytron of E. liassica Gardiner, 1961 shows none of the diagnostic characters of Elateridae. Carpenter [38] classified it in Coleoptera incertae sedis. The position of Fengningia Hong, 1984 (Cretaceous of China) [127] in Elateridae was questioned by Dong \& Huang [79], and this genus is transferred in this study to Coleoptera incertae sedis. The genus Macronotus Hong \& Wang, 1990 from the Cretaceous of China was originally placed in Buprestidae [139] but Dong \& Huang [79] suggested it might belong to Elateridae based on the shape of elytra. However, for example Ding et al. [140] 
and Kirejtshuk \& Ponomarenko [33] listed this genus again in Buprestidae. Three genera from the Triassic of Argentina, which were described based on the elytra only, i.e., Babuskaya, Cardiosyne and Gemelina [103,124], were originally classified in Elateridae only provisionally (with a question mark), and all of them are placed in Coleoptera incertae sedis in this study. Additionally, Dong et al. [87] listed in Elateridae Mesagyrtes Ponomarenko, 1977 from the Jurassic of the Russian Federation, although with a question mark. Mesagyrtes Ponomarenko, 1977 was preoccupied by Mesagyrtes Broun, 1895, and therefore, the former name was replaced by Mesecanus Newton, 1982. This genus is currently classified in the staphylinoid family Agyrtidae as already proposed in the original publication [141]. Another genus name proposed in Elateridae was Pseudoelater Heer, 1847 [97], but this is an unavailable name (nomen nudum) according to the Code [41], because no species was assigned to it.

The current composition of fossil Elateridae includes 74 extinct genera and three subgenera. Fifty-five genera are classified in eight subfamilies, and additional 19 ones have an uncertain position within Elateridae, and remain as incertae sedis. Almost half of genera are included in the only extinct subfamily, Protagrypninae, which consists of four tribes $[13,18,19,26,28]$. This subfamily is characterized by the unique prosternum and mesoventrite but is in need of revision; some species originally placed there by Dolin [13] were already moved to Cerophytidae, and some other need to be thoroughly re-examined [81,84]. Regarding the extant elaterid subfamilies, five most species-rich ones, which together form the bulk of the recent click-beetle diversity, are represented in fossil record by some extinct genera. These subfamilies are widely accepted and cosmopolitan Elaterinae, Dendrometrinae, Agrypninae, Cardiophorinae, and Negastriinae. However, the numbers of fossil genera assigned to extant subfamilies are not very high, and in some cases even their placement is debatable. This is not surprising, because the morphological limits of many elaterid subfamilies and tribes are uncertain and there is no reliable identification key to higher taxa which would be valid worldwide. Often, there are no clear synapomorphies available even for the apparently monophyletic groups defined by the molecular-based phylogenetic methods [1,9,10]. Moreover, many higher taxa are defined by the combination of characters including some which are usually not available for the fossils, such as the larval morphology, wing venation, etc., [4,9,78]. It is especially not easy to assign the compression fossils into a proper subfamily, and this is particularly true for the large subfamilies Elaterinae and Dendrometrinae, whose adults can be usually (but not always) recognized by the shape of fronto-clypeal region $[1,5]$. Maybe this is the reason why both above-mentioned subfamilies include mainly taxa described from the amber inclusions rather than the compressions [32]. Elaterinae includes five fossil genera and one subgenus, and Dendrometrinae includes two genera and two subgenera. However, the positions of some of them need to be re-examined. For example, the genus Cryptagriotes Wickham, 1916, described based on the compression fossil from the Eocene of the United States, was hypothesized to be similar either to Cryptohypnus Eschscholtz, 1830 (syn. of Hypnoidus Dillwyn, 1829 [69]) (Dendrometrinae: Hypnoidini) or Agriotes Eschscholtz, 1830 (Elaterinae: Agriotini) [29,38]. Another large subfamily, Agrypninae, is represented by seven genera classified in three tribes, one of which is a monogeneric fossil Cryptocardiini. Although Dolin \& Nel [89] placed another genus, Cryptocoelus, into this tribe, Chang et al. [56] later removed it from there, and also from Agrypninae, based on the different morphology of scutellum and prosternum. Three fossil genera usually classified in Cardiophorinae [13,20,61] were critically revised by Douglas [63,74]; two of them were removed from the subfamily, and the placement of the third one remains dubious. The closely related subfamily Negastriinae includes only two described fossil genera $[13,20]$. The smaller elaterid subfamilies, which include fossil genera, are represented by Omalisinae and Pityobiinae. The first one was placed into Elateridae only recently based on the molecular phylogeny [8], which explains why its only fossil genus, Jantarokrama, was earlier listed in Omalisidae [55,77].

The exact age of origin for Elateridae remains unclear, but there are many indications that they were already present in the Triassic Period $[12,14,15]$. Undescribed elaterids are reported from the Madygen Formation in southwest Kyrgyzstan, which dates back to Ladinian of Middle Triassic (242.0-228.0 Ma) [15,128]. The fossil record of Elateridae from the Triassic is somewhat problematic, 
because usually only body fragments, mostly elytra, are known for vast majority of species, and in the past, many doubtful specimens were designated as elaterids [35,93]. Genera Babuskaya, Cardiosyne, Gemelina (all from the Upper Triassic of Argentina, Carmian, 230 Ma), Mimelater, Plastelater, Stenelytron (all from the Upper Triassic of the United Kingdom, Rhaetian, 205 Ma), and Pseudoelateropsis (Triassic of Germany, Rhaetian, $205 \mathrm{Ma}$ ) cannot be classified in Elateridae with certainty, and so they are currently considered Coleoptera incertae sedis [38]. The Triassic record of Elateridae which is based on elytra only, needs further investigation and should be interpreted with caution. This includes Elaterium bipunctatum Dunstan, 1923 and all three species of Elateridium from the Triassic of Australia (Norian, 220 Ma and Anisian, $245 \mathrm{Ma}$ ), and Elaterophanes acutus Cockerell, 1915 from the Triassic of the United Kingdom (Rhaetian, $205 \mathrm{Ma}$ ) [86,96,100]. The only named representative of the Triassic Elateridae, for which we have habitus information and not only a single elytron available, is Elaterophanes vetustus (Brodie, 1845) from the Rhaetian Lilstock Formation in England (208.5-201.3 Ma) [12,35,85]. While Triassic Elaterium and Elateridium cannot be reliably placed in any of the elaterid subfamilies, Elaterophanes has been classified in Protagrypninae [14]. This fossil subfamily became increasingly abundant and diverse later in the Middle Jurassic, as documented by the presence of almost 30 genera classified in the tribes Desmatini, Hypnomorphini, and Protagrypnini [13,14,17,19,21,22,79,91]. Some other lineages of Elateridae also diversified during the Jurassic Period, such as Agrypninae (Agrypnini, Cryptocardiini), Dendrometrinae (Dimini), and Negastriinae [13,20]. The diversification of elaterids continued through the Cretaceous Period [23-28,89,95], and many recent genera were established by the early Cenozoic $[16,29,33,142]$. It is remarkable that no genera of Elaterinae and Dendrometrinae, which are recently the most genus-rich subfamilies, have not been reported from the Mesozoic. The only exception is Alaodima, which is assigned to Dimini, but there is an ongoing debate if this group belongs to Dendrometrinae or forms a separate subfamily [13,72]. The missing record of Elaterinae and Dendrometrinae genera in the early evolution of Elateridae is most probably artificial, because they belong to the basal-most splits based on the results of recent molecular phylogenetic analyses [9-11]. This situation may be partly caused by the hard-to-observe morphological characters defining the subfamilies and their tribes $[1,4,5,78]$, which are usually not visible even if the fossils are well-preserved. In badly preserved click-beetle fossils, it is often almost impossible to find any important diagnostic character which would allow researchers to classify it correctly to the higher taxonomic rank. Another problem might be the misidentifications of some described taxa and misinterpretations of their systematic placements, and also the general underexamination of the fossil record, especially from the Mesozoic amber deposits [16,27,128]. Genera which are currently considered incertae sedis in Elateridae should be carefully investigated in order to improve our knowledge on the palaeodiversity of the internal lineages in Elateridae, including Elaterinae and Dendrometrinae.

\section{Conclusions}

The systematics and classification of fossil Elateridae is in a state of flux. Recent increase in publications focusing on the extinct click-beetles brought many new discoveries [25-28,77,79], which sounds promising for future research in the group. However, much more attempt should be made to better explore the real palaeodiversity of Elateridae. First of all, the revisions of taxa on all taxonomic levels should be made, including the critical re-examination of the only fossil subfamily Protagrypninae, which currently includes by far the highest generic diversity in fossil click-beetles $[13,81,84]$. These revisions should also include the incertae sedis taxa, which include mainly lineages from the early evolution of Elateridae in the Mesozoic Era. Additionally, the so-far unnamed click-beetle fossils reported from various deposits $[15,16,55,128]$ should be investigated in order to help us better understand the real diversity and estimate the origin of main click-beetle lineages. For example, the amber deposits (especially Baltic, Dominican, Lebanese, and Burmese) are highly understudied and may be of a great importance because of the much better preservation of specimens than in the case of compression fossils $[16,27,39,55,128]$. Recent revision of the fossil representatives of the morphologically similar family Cerophytidae revealed much greater diversity than previously 
believed [94], and this can be expected also in Elateridae. Our study of the fossil genus-group name concepts in Elateridae should serve future scholars as a robust framework for further research on the palaeodiversity and evolution of the click-beetles.

Author Contributions: Conceptualization, R.K.; investigation, R.K., G.P., and J.H.; writing-original draft, R.K.; supervision, R.K.; project administration, R.K.; writing—review and editing, R.K., G.P., and J.H.; funding acquisition, R.K. All authors have read and agreed to the published version of the manuscript.

Funding: This research was funded by the internal grant of the Palacky University nr. IGA_PrF_2020_026.

Acknowledgments: We would like to express our sincere gratitude to the following colleagues for providing literature: David Peris (Germany), Alexander S. Prosvirov (Russia), Karol Szawaryn (Poland), Andris Bukejs (Latvia), John F. Lawrence (Australia), Christel Hoffeins (Germany), Christoph Schindler (Germany), Erik Tihelka (Great Britain), Neal Evenhuis (USA), and Oscar F. Gallego (Argentina). Further, we thank Miguel A. Alonso-Zarazaga (Spain) for his invaluable help with identification of correct gender of some genus-group names.

Conflicts of Interest: The authors declare no conflict of interest. The funders had no role in the design of the study; in the collection, analyses, or interpretation of data; in the writing of the manuscript, or in the decision to publish the results.

\section{References}

1. Costa, C.; Lawrence, J.F.; Rosa, S.P. Elateridae Leach, 1815. In Handbook of Zoology, Arthropoda: Insecta; Coleoptera, Beetles; Volume 2: Morphology and Systematics (Elateroidea, Bostrichiformia, Cucuijormia Partim); Leschen, R.A.B., Beutel, R.G., Lawrence, J.F., Eds.; Walter de Gruyter GmbH \& Co. KG: Berlin, Germany; New York, NY, USA, 2010; pp. 75-103.

2. Traugott, M.; Benefer, C.M.; Blackshaw, R.P.; van Herk, W.G.; Vernon, R.S. Biology, ecology, and control of elaterid beetles in agricultural land. Annu. Rev. Entomol. 2015, 60, 313-334. [CrossRef] [PubMed]

3. Schwarz, O. Coleoptera. Fam. Elateridae. In Genera Insectorum; Fascicules 46A, 46B; Wytsman, P.A., Ed.; P. Wytsman: Brussels, Belgium, 1906; Volume VII, pp. 1-224.

4. Dolin, V.G. Wing venation in click-beetles (Coleptera, Elateridae) and its significance in the taxonomy of the family. Zool. Zhurnal 1975, 54, 1618-1633.

5. Stibick, J.N.L. Classification of the Elateridae (Coleoptera). Relationships and classification of the subfamilies and tribes. Pac. Insects 1979, 20, 145-186.

6. Calder, A.A.; Lawrence, J.F.; Trueman, J.W.H. Austrelater, gen. nov. (Coleoptera: Elateridae), with a description of the larva and comments on elaterid relationships. Invertebr. Taxon. 1993, 7, 1349-1394. [CrossRef]

7. Kundrata, R.; Bocak, L. The phylogeny and limits of Elateridae (Insecta, Coleoptera): Is there a common tendency of click beetles to soft-bodiedness and neoteny? Zool. Scr. 2011, 40, 364-378. [CrossRef]

8. Kusy, D.; Motyka, M.; Bocek, M.; Vogler, A.P.; Bocak, L. Genome sequences identify three families of Coleoptera as morphologically derived click beetles (Elateridae). Sci. Rep. 2018, 8, 17084. [CrossRef]

9. Kundrata, R.; Gunter, N.L.; Janosikova, D.; Bocak, L. Molecular evidence for the subfamilial status of Tetralobinae (Coleoptera: Elateridae), with comments on parallel evolution of some phenotypic characters. Arthropod Syst. Phyl. 2018, 76, 137-145.

10. Kundrata, R.; Prosvirov, A.S.; Vondracek, D.; Sormova, E. Congruence between molecular data and morphology: Phylogenetic position of Senodoniini (Coleoptera: Elateridae). Insects 2019, 10, 231. [CrossRef]

11. Bi, W.-X.; He, J.-W.; Chen, C.-C.; Kundrata, R.; Li, X.-Y. Sinopyrophorinae, a new subfamily of Elateridae (Coleoptera, Elateroidea), with the first record of a luminous click beetle in Asia and evidence for multiple origins of bioluminescence in Elateridae. ZooKeys 2019, 864, 79-97. [CrossRef]

12. Brodie, P.B. A History of the Fossil Insects in the Secondary Rocks of England Accompanied by a Particular Account of the Strata in which they Occur, and of the Circumstances Connected with their Preservation; John Van Voorst: London, UK, 1845; pp. 1-130.

13. Dolin, V.G. Click beetles (Coleoptera, Elateridae) from the Upper Jurassic of Karatau. In Fossil Insects of the Mesozoic; Dolin, V.G., Panfilov, D.V., Ponomarenko, A.G., Pritykina, D.N., Eds.; Naukova Dumka: Kiev, Ukraine, 1980; pp. 17-81. 
14. Whalley, P.E.S. The systematics and palaeogeography of the Lower Jurassic insects of Dorset, England. Bull. Br. Mus. Nat. Hist. Geol. 1985, 39, 107-189.

15. Ponomarenko, A.G. The geological history of beetles. In Biology, Phylogeny and Classification of Coleoptera: Papers Celebrating the 80th Birthday of Roy A. Crowson; Pakaluk, J., Ślipiński, S.A., Eds.; Muzeum i Instytut Zoologii PAN: Warszawa, Poland, 1995; pp. 87-155.

16. Grimaldi, D.; Engel, M. Evolution of the Insects; Cambridge University Press: New York, NY, USA; Cambridge, UK, 2005; pp. 1-755.

17. Chang, H.L.; Kirejtshuk, A.G.; Ren, D. First fossil click beetles from the Middle Jurassic of Inner Mongolia, China (Coleoptera: Elateridae). Ann. Zool. 2009, 59, 7-14. [CrossRef]

18. Dolin, V.G. Iskopaemye formy Zhukov-Shchelkunov (Elateridae, Coleoptera) iz Nizhney Yury Sredney Azii. In Fauna i Biologiya Nasekomykh Moldavii, III; Yaroshenko, M., Ed.; Shtiintsa: Kishinev, Moldova, 1973; pp. 71-82.

19. Dolin, V.G. A contribution to the systematics of Mesozoic click beetles (Coleoptera, Elateridae). Paleontol. J. 1975, 9, 474-486.

20. Dolin, V.G. Fossil click beetles (Coleoptera, Elateridae) of the subfamilies Negastriinae and Cardophorinae from Upper Jurassic of Karatau. Vestn. Zool. 1976, 1976, 68-75.

21. Lin, Q.B. Early Mesozoic fossil insects from South China. Palaeontol. Sinica, Ser. B 1986, 170, 1-112.

22. Dong, F.B.; Huang, D.Y. A new click beetle (Coleoptera: Elateridae) from Middle Jurassic Haifanggou Formation of western Liaoning, China. Acta Palaeontol. Sinica 2009, 48, 102-108.

23. Ping, C. Study of the Cretaceous fossil insects of China. Palaeontol. Sinica, Ser. B 1928, 13, 5-7.

24. Chang, H.L.; Ren, D. New fossil beetles of the family Elateridae from the Jehol Biota of China (Coleoptera: Polyphaga). Acta Geol. Sinica 2008, 82, 236-243. [CrossRef]

25. Chang, H.L.; Kirejtshuk, A.; Ren, D. New fossil elaterids (Coleoptera: Polyphaga: Elateridae) from the Jehol biota in China. Ann. Entomol. Soc. Am. 2010, 103, 866-874. [CrossRef]

26. Alekseev, A.V. New click beetles (Coleoptera: Elateridae) from the Cretaceous of Russia and Kazakhstan. Paleontol. J. 2011, 45, 423-431. [CrossRef]

27. Otto, R.L. Descriptions of two new elateroid beetles (Coleoptera: Eucnemidae, Elateridae) from Burmese amber. Insecta Mundi 2019, 702, 1-6. [CrossRef]

28. Sohn, J.C.; Nam, G.S.; Choi, S.W.; Ren, D. New fossils of Elateridae (Insecta, Coleoptera) from Early Cretaceous Jinju Formation (South Korea) with their implications to evolutionary diversity of extinct Protagrypninae. PLoS ONE 2019, 14, e0225502. [CrossRef] [PubMed]

29. Wickham, H.F. The fossil Elateridae of Florissant. Bull. Mus. Comp. Zool. 1916, 60, 493-527.

30. Haupt, H. Die Käfer (Coleoptera) aus der eozänen Braunkohle des Geiseltales. Geologica 1950, 6, 1-168.

31. Tröster, G. Eine neue Gattung der Elateridae (Insecta: Coleoptera) Macropunctum gen. n. aus der Messel-Formation des unteren Mittel-Eozän der Fundstätte Messel. Cour. Forschungsinst. Senckenberg 1991, 139, 99-117.

32. Iablokoff-Khnzorian, S.M. Novye zhestkokrylye semeystva Elateridae iz baltiyskogo yantayra. Paleontol. Zh. 1961, 1961, 84-97.

33. Kirejtshuk, A.G.; Ponomarenko, A.G. Taxonomical list of fossil beetles of the suborder Scarabaeina (part 2). [last update: July 2015]. Available online: https://www.zin.ru/Animalia/Coleoptera/eng/paleosy1.htm (accessed on 10 May 2020). (In Russian).

34. Scudder, S.H. Index to the Known Fossil Insects of the World including Myriapods and Arachnids; US Government Printing Office: Washington, DC, USA, 1891; Volume 71, pp. 1-744.

35. Handlirsch, A. Die Fossilen Insekten und die Phylogenie der Rezenten Formen, parts I-IV. Ein Handbuch fur Palaontologen und Zoologen; Wilhelm Engelmann: Leipzig, Germany, 1906; pp. 1-640.

36. Hyslop, J.A. Genotypes of the Elaterid beetles of the world. Proc. U. S. Nat. Mus. 1921, 58, $621-673$. [CrossRef]

37. Spahr, U. Systematischer Katalog der Bernstein- und Kopal-Käfer (Coleoptera). Systematic catalogue of Coleoptera in amber and copal. Stuttg. Beitr. Naturkd. Ser. B 1981, 80, 1-107.

38. Carpenter, F.M. Volume 4: Superclass Hexapoda. In Treatise on Invertebrate Paleontology, Part R, Arthropoda 4; Kaesler, R.D., Ed.; Geological Society of America: Boulder, Colorado; University of Kansas: Lawrence, KS, USA, 1992; pp. 279-655. 
39. Alekseev, V.I. The beetles (Insecta: Coleoptera) of Baltic amber: The checklist of described species and preliminary analysis of biodiversity. Zool. Ecol. 2013, 23, 5-12. [CrossRef]

40. Kundrata, R.; Kubaczkova, M.; Prosvirov, A.S.; Douglas, H.B.; Fojtikova, A.; Costa, C.; Bousquet, Y.; Alonso-Zarazaga, M.A.; Bouchard, P. World catalogue of the genus-group names in Elateridae (Insecta, Coleoptera). Part I: Agrypninae, Campyloxeninae, Hemiopinae, Lissominae, Oestodinae, Parablacinae, Physodactylinae, Pityobiinae, Subprotelaterinae, Tetralobinae. ZooKeys 2019, 839, 83-154. [CrossRef]

41. ICZN. International Commission on Zoological Nomenclature. In International Code of Zoological Nomenclature. Fourth Edition; The International Trust for Zoological Nomenclature: London, UK, 1999; pp. 1-305.

42. Cohen, K.M.; Finney, S.C.; Gibbard, P.L.; Fan, J.X. The ICS International Chronostratigraphic Chart. Episodes 2013, 36, 199-204. [CrossRef]

43. Leach, W.E. Entomology. In Edinburgh Encyclopaedia, Volume IX, Part I; Brewster, D., Blackwood, W., Waugh, J., Eds.; William Blackwood etc.: Edinburgh, UK, 1815; pp. 57-172.

44. Bouchard, P.; Bousquet, Y.; Davies, A.E.; Alonso-Zarazaga, M.A.; Lawrence, J.F.; Lyal, C.H.C.; Newton, A.F.; Reid, C.A.M.; Schmitt, M.; Ślipiński, S.A.; et al. Family-group names in Coleoptera (Insecta). ZooKeys 2011, 88, 1-972. [CrossRef] [PubMed]

45. Candèze, E.C.A. Monographie des Élatérides. Tome premier. Mem. Soc. R. Sci. Liege 1857, 12, 1-400.

46. Korneev, V.A.; Cate, P. Names of insects described by Vladimir Gdalich Dolin. Vestn. Zool. 2005, 39, 9-26.

47. Tröster, G. Zur Kenntnis der Gattung Macropunctum (Elateridae, Pyrophorinae, Agrypnini) aus der mitteleozänen Fossillagerstätte “Eckfelder Maar" (Eifel) mit der Beschreibung einer neuen Art Macropunctum eckfeldi n. sp. Mainzer Naturwiss. Archiv 1992, 30, 111-118.

48. Tröster, G. Fossile Schnellkäfer der Gattung Lanelater Arnett 1952 (Coleoptera, Pyrophorinae, Agrypnini) aus dem Eozän der Grube Messel bei Darmstadt. Senck Leth 1993, 73, 49-60.

49. Tröster, G. Fossile Elateridae (Insecta: Coleoptera) aus dem Unteren Mitteleozän (Lutetium) der Grube Messel bei Darmstadt. Cour. Forschungsinst. Senckenberg 1994, 170, 11-64.

50. Tröster, G. Neue Arten der Gattung Macropunctum (Insecta, Coleoptera, Elateridae) aus der Olschieferfazies der mitteleozänen Messelformation der Grube Messel bei Darmstadt. Palaontol. Z. 1994, 68, 145-162. [CrossRef]

51. Tröster, G. An unusual new fossil click-beetle (Coleoptera: Elateridae) from the Middle Eocene of the Grube Messel (Germany). N. Jb. Geol. Palaeont. Mh. 1999, 1999, 11-20. [CrossRef]

52. Wappler, T. Systematik, Phylogenie, Taphonomie und Paläoökologie der Insekten aus dem Mittel-Eozän des Eckfelder Maares, Vulkaneifel. Clausthal. Geowissensch. 2003, 2, 1-241.

53. Kirejtshuk, A.G.; Ponomarenko, A.G.; Kurochkin, A.S.; Alekseev, A.V.; Gratshev, V.G.; Solodovnikov, A.V.; Krell, F.-T.; Soriano, C. The beetle (Coleoptera) fauna of the Insect Limestone (late Eocene), Isle of Wight, southern England. Earth Environm. Sci. Trans. R. Soc. Edinburgh 2019, 110, 405-492. [CrossRef]

54. Larsson, S.G. Baltic amber-A palaeobiological study. Entomonograph 1978, 1, 1-192.

55. Alekseev, V.I. Coleoptera from the middle-upper Eocene European ambers: Generic composition, zoogeography and climatic implications. Zootaxa 2017, 4290, 401-443. [CrossRef]

56. Chang, H.L.; Ren, D.; Shih, C.K. New fossil elaterid (Coleoptera: Polyphaga: Elateridae) from Yixian Formation of western Liaoning, China. Prog. Nat. Sci. 2007, 17, 1244-1249.

57. Candèze, E.C.A. Monographie des Élatérides. Tome quatrieme. Mem. Soc. R. Sci. Liege 1863, 17, 1-534.

58. Haupt, H. Beitrag zur Kenntnis der eozänen Arthopodenfauna des Geiseltales. Nova Acta Leopold. 1956, $18,1-89$.

59. Kinzelbach, R.K.; Lutz, H. Stylopid larva from the Eocene-A spotlight on the phylogeny of the Stylopids (Strepsiptera). Ann. Entomol. Soc. Am. 1985, 78, 600-602. [CrossRef]

60. Candèze, E.C.A. Monographie des Élatérides. Tome second. Mem. Soc. R. Sci. Liege 1859, 14, 1-543.

61. Becker, E.C. Three new fossil elaterids from the amber of Chiapas, Mexico, including a new genus (Coleoptera). J. Paleontol. 1963, 37, 125-128.

62. Solórzano Kraemer, M.M. Systematic, palaeoecology, and palaeobiogeography of the insect fauna from Mexican amber. Palaeontogr. Abt. A 2007, 282, 1-133. [CrossRef]

63. Douglas, H. World reclassification of the Cardiophorinae (Coleoptera, Elateridae), based on phylogenetic analyses of morphological characters. ZooKeys 2017, 655, 1-130. [CrossRef]

64. Gistel, J.N.F.X. Faunula monacensis cantharologica. Isis von Oken 1848, 1848, 4-6.

65. Eschscholtz, J.F. Elaterites, Eintheilung derselben in Gattungen. Entomol. Arch. 1829, 2, 31-35. 
66. Fabricius, J.C. Entomologia Systematica Emendata et Aucta. Secundum Classes, Ordines, Genera, Species Adjectis Synonimis, Locis, Observationibus, Descriptionibus; Christian Gottlob: Copenhagen, Denmark, 1792; pp. 1-330.

67. Westwood, J.O. An introduction to the modern classification of insects, founded on the natural habits and corresponding organization of the different families. In Synopsis of the Genera of British Insects; Longman, Orme, Brown, Green \& Longmans: London, UK, 1838; Volume II, pp. 1-48.

68. Sánchez-Ruiz, A. Catálogo bibliográfico de las especies de la familia Elateridae (Coleoptera) de la Península Ibérica e Islas Baleares. In Documentos Fauna Ibérica, 2; Ramos, M.A., Ed.; Museo Nacional de Ciencias Naturales, CSIC: Madrid, Spain, 1996; pp. 1-265.

69. Cate, P.C. Elateridae Leach, 1815 (-Cebrioninae, Lissominae, Subprotelaterinae). In Catalogue of Palaearctic Coleoptera; Löbl, I., Smetana, A., Eds.; Apollo Books: Stenstrup, Denmark, 2007; Volume 4, pp. 89-209.

70. Linnaeus, C. Systema Naturae Per Regna Tria Naturae: Secundum Classes, Ordines, Genera, Species, Cum Characteribus, Differentiis, Synonymis, Locis. Tomus I, Editio Decima, Reformata; Laurentius Salvius: Stockholm, Sweden, 1758; pp. 1-823.

71. Curtis, J. British Entomology, being Illustrations and Descriptions of the Genera of Insects Found in Great Britain and Ireland: Containing Coloured Figures from Nature of the Most Rare and Beautiful Species, and in Many Instances of the Plants upon which they are Found; J. Curtis: London, UK, 1838; Volume XV, pp. 674-721.

72. Kundrata, R.; Musalkova, M.; Kubaczkova, M. Annotated catalogue of the click-beetle tribe Dimini (Coleoptera: Elateridae: Dendrometrinae). Zootaxa 2018, 4412, 1-75. [CrossRef]

73. Dejean, P.F.M.A. Catalogue des Coléoptères de la Collection de M. le Comte Dejean; Livraisons 1-2; Méquignon-Marvis: Paris, France, 1833; pp. 1-176.

74. Douglas, H. Phylogenetic relationships of Elateridae inferred from adult morphology, with special reference to the position of Cardiophorinae. Zootaxa 2011, 2900, 1-45. [CrossRef]

75. Nakane, T.; Kishii, T. On the subfamilies of Elateridae from Japan (Coleoptera). Kontŷิ 1956, 24, $201-206$.

76. Lacordaire, J.T. Histoire Naturelle des Insectes. Genera des Coléoptères ou Exposé Méthodique et Critique de Tous les Genres Proposés Jusqu'ici dans cet Ordre d'insectes. Tome Quatrième Contenant les Familles des Buprestides, Throscides, Eucnémides, Élatérides, Cébrionides, Cérophytides, Rhipicérides, Dascyllides, Malacodermes, Clérides, Lyméxylones, Cupésides, Ptiniores, Bostrichides et Cissides; Librairie Encyclopédique de Roret: Paris, France, 1857; pp. 1-579.

77. Kirejtshuk, A.G.; Kovalev, A.V. First fossil representative of the family Omalisidae (Coleoptera, Elateroidea sensu lato) from the Baltic amber. Paleontol. J. 2015, 49, 1413-1416. [CrossRef]

78. Hyslop, J.A. The phylogeny of the Elateridae based on larval characters. Ann. Entomol. Soc. Am. 1917, 10, 241-263. [CrossRef]

79. Dong, F.B.; Huang, D.Y. A new elaterid from the Middle Jurassic Daohugou biota (Coleoptera: Elateridae: Protagrypninae). Acta Geol. Sinica 2011, 85, 1224-1230.

80. Yu, Y.; Liu, Z.H.; Shih, C.H.; Ren, D. Coleoptera-Beetles. In Rhythms of Insect Evolution: Evidence from the Jurassic and Cretaceous in Northern China, 1st ed.; Ren, D., Shih, C.H., Gao, T., Yao, Y.U., Wang, Y., Eds.; John Wiley \& Sons, Ltd.: Hoboken, NJ, USA, 2019; pp. 337-428.

81. Chang, H.L.; Zhang, F.; Ren, D. A new genus and two new species of fossil Elaterids from the Yixian Formation of western Liaoning, China (Coleoptera: Elateridae). Zootaxa 2008, 1785, 54-62. [CrossRef]

82. Kirejtshuk, A.G.; Ponomarenko, A.G.; Prokin, A.A.; Chang, H.; Nikolajev, G.V.; Ren, D. Current knowledge on Mesozoic Coleoptera from Daohugou and Liaoning (Northeast China). Acta Geol. Sinica 2010, 84, 783-792. [CrossRef]

83. Ponomarenko, A.G.; Yan, E.V.; Wang, B.; Zhang, H.C. Revision of some early Mesozoic beetles from China. Acta Palaeontol. Sinica 2012, 51, 475-490.

84. Chang, H.; Kirejtshuk, A.G.; Ren, D. On taxonomy and distribution of fossil Cerophytidae (Coleoptera: Elateriformia) with description of a new Mesozoic species of Necromera Martynov, 1926. Ann. Soc. Entomol. Fr. 2011, 47, 33-44. [CrossRef]

85. Giebel, C.G. Fauna der Vorwelt Mit steter Berücksichtigung der Lebenden Thiere. Monographisch Dargestellt. Zweiter Band: Gliederthiere. Erste Abtheilung. Insecten und Spinnen; Brockhaus: Leipzig, Germany, 1856; pp. 1-511.

86. Cockerell, T.D.A. British fossil insects. Proc. U. S. Nat. Mus. 1915, 49, 469-499. [CrossRef]

87. Dong, F.B.; Cai, C.Y.; Huang, D.Y. Revision of five Mesozoic beetles from southern China. Acta Palaeontol. Sinica 2011, 50, 481-491. 
88. Zhang, H.C. A new genus of Elateridae (Insecta, Coleoptera) from Lower-Middle Jurassic of Junggar Basin, Xinjiang, China. Acta Micropalaeontol. Sinica 1997, 14, 71-77.

89. Dolin, V.G.; Nel, A. Trois nouveaux Elateridae fossiles du Mésozoïque supérieur de Chine (Coleoptera). Bull. Soc. Entomol. Fr. 2002, 107, 341-346.

90. Martin, S.K. Early Jurassic coleopterans from the Mintaja insect locality, Western Australia. Acta Geol. Sinica 2010, 84, 925-953. [CrossRef]

91. Chang, H.L.; Zhao, Y.Y.; Ren, D. New fossil elaterids (Insect: Coleoptera: Polyphaga: Elateridae) from the Middle Jurassic of Inner Mongolia, China. Prog. Nat. Sci. 2009, 19, 1433-1437. [CrossRef]

92. Scudder, S.H. Adephagous and clavicorn Coleoptera from the Tertiary deposits at Florissant, Colorado with descriptions of a few other forms and a systematic list of the non-rhynchophorus Tertiary Coleoptera of North America. Monogr. U. S. Geol. Surv. 1900, 40, 1-148.

93. Handlirsch, A. Die Fossilen Insekten und die Phylogenie der Rezenten Formen, part V. Ein Handbuch fur Palaontologen und Zoologen; Wilhelm Engelmann: Leipzig, Germany, 1907; pp. 641-800.

94. Yu, Y.; Ślipiński, A.; Lawrence, J.F.; Yan, E.; Ren, D.; Pang, H. Reconciling past and present: Mesozoic fossil record and a new phylogeny of the family Cerophytidae (Coleoptera: Elateroidea). Cret. Res. 2019, 99, 51-70. [CrossRef]

95. Alekseev, A.V. New Jewel Beetles (Coleoptera: Buprestidae) and Similar Beetles from the Cretaceous and Early Paleogene of Asia. Paleontol. J. 2008, 42, 53-59. [CrossRef]

96. Tillyard, R.J. Mesozoic and Tertiary Insects of Queensland and New South Wales. Descriptions of the fossil Insects. Qld. Geol. Surv. Pub. 1916, 253, 11-70.

97. Heer, O. Die Insektenfauna der Tertiärgebilde von Oeningen und von Radoboj in Croatien. Erster Theil: Käfer; Wilhelm Engelmann: Leipzig, Germany, 1847; pp. 1-229.

98. Tillyard, R.J. Permian and Triassic insects from New South Wales, in the collection of Mr. John Mitchell. Proc. Linn. Soc. N. S. W. 1918, 42, 720-756.

99. Ponomarenko, A.G. New beetles (Insecta, Coleoptera) from Vyazniki Locality, terminal Permian of European Russia. Paleontol. J. 2011, 45, 414-422. [CrossRef]

100. Dunstan, B. Mesozoic Insects of Queensland Part I.-Introduction and Coleoptera. Qld. Geol. Surv. Pub. 1923, 273, 1-89.

101. Handlirsch, A. Neue Untersuchungen über die fossilen Insekten mit Ergänzungen und Nachträgen sowie Ausblicken auf phylogenetische, palaeogeographische und allgemein biologische Probleme. II. Teil. Ann. Naturhist. Mus. Wien. 1938, 49, 1-240.

102. Jell, P.A. The fossil insects of Australia. Mem. Queensland Mus. 2004, 50, 1-124.

103. Martins-Neto, R.G.; Gallego, O.F.; Mancuso, A.C. The Triassic insect fauna from Argentina. Coleoptera from Los Rastros Formation (Bermejo Basin), La Rioja Province. Ameghiniana 2006, 43, 591-609.

104. Deichmüller, J.V. Fossile Insecten aus dem Diatomeenschiefer von Kutschlin bei Bilin, Böhmen. Nova Acta Acad. Caes. Leop.-Carol. German. Nat. Cur. 1881, 42, 293-330.

105. Scudder, S.H. 4. Classe Insecta. Insecten. In Handbuch der Palaeontologie, I Abtheilung. Palaeozoologie 2; Zittel, K., Ed.; Oldenbourg: München/Leipzig, Germany, 1885; pp. 747-831.

106. Scudder, S.H. Systematic Review of our Present Knowledge of Fossil Insects including Myriapods and Arachnids; Government Printing Office: Washington, DC, USA, 1886; pp. 1-128.

107. Cockerell, T.D.A. Fossil Arthropods in the British Museum, III. Ann. Mag. Nat. Hist. 1920, 5, 455-463. [CrossRef]

108. Cockerell, T.D.A. Some Tertiary fossil insects. Ann. Mag. Nat. Hist. 1926, 18, 313-324. [CrossRef]

109. Westwood, J.O. Contributions to fossil entomology. Quart. J. Geol. Soc. London 1854, 10, 378-396. [CrossRef]

110. Coram, R.A.; Jepson, J.E. Fossil Insects of the Purbeck Limestone Group of Southern England: Palaeoentomology from the Dawn of the Cretaceous; Siri Scientific Press: Manchester, UK, 2012; pp. 1-144.

111. Kirejtshuk, A.G. Taxonomic Review of Fossil Coleopterous Families (Insecta, Coleoptera). Suborder Archostemata: Superfamilies Coleopseoidea and Cupedoidea. Geosciences 2020, 10, 73. [CrossRef]

112. Chang, H.; Kirejtshuk, A.G.; Ren, D. New genus and species of Cerophytidae (Insecta: Coleoptera) from the Lower Cretaceous of Baissa (Siberia). Cret. Res. 2011, 32, 700-704. [CrossRef]

113. Oberprieler, R.G.; Ashman, L.G.; Frese, M.; Ślipiński, A. The first elateroid beetles Coleoptera: Polyphaga: Elateroidea) from the Upper Jurassic of Australia. Zootaxa 2016, 4147, 177-191. [CrossRef] [PubMed] 
114. Kundrata, R.; Jäch, M. Ptilodactyla crenatostriata Redtenbacher, 1868 (Coleoptera: Ptilodactylidae) transferred to Phytocerum Costa, Vanin, Lawrence \& Ide, 2003 (Coleoptera: Cerophytidae). Zootaxa 2017, 4324, 371-377. [CrossRef]

115. Yan, E.V.; Zhang, H. New beetle species of the formal genus Artematopodites (Coleoptera: Polyphaga), with remarks on the taxonomic position of the genera Ovivagina and Sinonitidulina. Paleontol. J. 2010, 44, 451-456. [CrossRef]

116. Yan, E.V.; Wang, B.; Zhang, H. First record of the beetle family Lasiosynidae (Insecta: Coleoptera) from the Lower Cretaceous of China. Cret. Res. 2013, 40, 43-50. [CrossRef]

117. Kirejtshuk, A.G.; Azar, D. Current knowledge of Coleoptera (Insecta) from the Lower Cretaceous Lebanese amber and taxonomical notes for some Mesozoic groups. Terr. Arthropod Rev. 2013, 6, 103-134. [CrossRef]

118. Yan, E.V.; Wang, B.; Zhang, H. A new lasiosynid beetle from the Middle Jurassic of China with remarks on the systematic position of Lasiosynidae. C. R. Palevol 2014, 13, 1-8. [CrossRef]

119. Ponomarenko, A.G. New beetles (Insecta, Coleoptera) from the Nedubrovo locality, terminal Permian or basal Triassic of European Russia. Paleontol. J. 2015, 49, 39-50. [CrossRef]

120. Heyden, C.V. Fossile Insekten aus der Rheinischen Braunkhohle. Palaeontographica 1859, 8, 1-15.

121. Crowson, R.A. The evolutionary history of Coleoptera, as documented by fossil and comparative evidence. In Proceedings of the Atti del X Congresso Nazionale Italiano di Entomologia, Sassari, Italy, 20-25 May 1974; pp. 47-90.

122. Hörnschemeyer, T. New species of Electribius Crowson 1973 (Coleoptera: Artematopodidae) from Baltic amber. Paläontol. Z. 1998, 72, 299-306. [CrossRef]

123. Zherikhin, V.V. Klass Insecta. Nasekomye. In Razvitie i Smena Bespozvonochnykh na Rubezhe Mesozoya i Kainozoya. Mshanki, Chlenistonogie i Glokozhie; Simanskij, V.N., Solovev, A.N., Eds.; Akademyia Nauk: Moscow, Russia, 1980; pp. 40-97.

124. Martins-Neto, R.G.; Gallego, O.F. The Triassic insect fauna from Argentina. Blattoptera and Coleoptera from the Ischichuca Formation (Bermejo Basin), La Rioja Province. Ameghiniana 2009, 46, 361-372.

125. Martins-Neto, R.G.; Gallego, O.F.; Tassi, L.V. The Triassic coleopteran fauna of southern South America: Morphometric variation of elytra, paleobiogeography, and a phylogenetic approach. GAEA 2011, 7, 1-18. [CrossRef]

126. Lara, M.B.; Gallego, O.F.; Tassi, L.V. Mesozoic coleopteran faunas from Argentina: Geological context, diversity, taphonomic observations, and comparison with other fossil insect records. Psyche 2012, 2012, 1-14. [CrossRef]

127. Hong, Y.C. Insecta. In Palaeontological Atlas of North China. Part II: Mesozoic; Yang, Y., Ed.; Geological Publishing House: Beijing, China, 1984; pp. 128-185.

128. Rasnitsyn, A.P.; Quicke, D.L.J. History of Insects; Kluwer Academic Publishers: Dordrecht, The Netherlands, 2002; pp. 1-517.

129. Nabozhenko, M.V. The Fossil Record of Darkling Beetles (Insecta: Coleoptera: Tenebrionidae). Geosciences 2019, 9, 514. [CrossRef]

130. Pongrácz, A. Die eozäne Insektenfauna des Geiseltales. Nova Acta Leopold. 1935, 2, 485-572.

131. Bellamy, C.L. World catalogue and bibliography of the jewel beetles (Coleoptera: Buprestoidea); Volume 1. Introduction; Fossil taxa; Schizopodidae; Buprestidae: Julodinae-Chrysochroinae: Poecilonotini; Pensoft Publishers: Sofia, Bulgaria, 2008; pp. 1-625.

132. Alekseev, A.V. Jurassic and Lower Cretaceous Buprestidae (Coleoptera) from Eurasia. Paleontol. J. 1993, 27, 9-34.

133. Ponomarenko, A.G. Zhestkokrylye iz Yury Sibiri i zapadnoy Mongolii. In Yurskie Nasekomye Sibiri i Mongolii; Rasnitsyn, A.P., Ed. Trudy Paleontol. Inst. Akad. Nauk SSSR 1985, 211, 47-87.

134. Ponomarenko, A.G. Systematic position of some beetles from the Solenhofen shales of Bavaria. Paleontol. J. 1971, 5, 62-75.

135. Hayek, C.M.F. A reclassification of the subfamily Agrypninae (Coleoptera: Elateridae). Bull. Brit. Mus. (Nat. Hist.) Entomol. 1973, 20, 1-309.

136. Martynov, A.V. K Poznaniyu Iskopaemykh Nasekomykh Yurskikh Slantsev Turkestana. 5. O Nekotorykh Formakh Zhukov (Coleoptera). Ezheg. Russ. Paleontol. Obsh. 1926, 5, 1-39.

137. Ponomarenko, A.G. Istoricheskoe Razvitie Zhestkokrylykh-Arkhostemat. Trudy Akademiya Nauk SSSR 1969, 125, 1-240. 
138. Ponomarenko, A.G. On the Types of Mesozoic Archostematan Beetles (Insecta, Coleoptera, Archostemata) in the Natural History Museum, London. Paleontol. J. 2006, 40, 90-99. [CrossRef]

139. Hong, Y.C.; Wang, W.L. Fossil insects from the Laiyang Basin, Shandong Province. In Stratigraphy and Palaeontology of Laiyang Basin, Shandong Province; Regional Geological Surveying Team, Shandong Bureau of Geology and Mineral Resources, Eds.; Geological Publishing House: Beijing, China, 1990; pp. 44-189.

140. Ding, Q.; Labandeira, C.C.; Ren, D. Biology of a leaf miner (Coleoptera) on Liaoningocladus boii (Coniferales) from the Early Cretaceous of northeastern China and the leaf-mining biology of possible insect culprit clades. Arthropod Syst. Phyl. 2014, 72, 281-308.

141. Ponomarenko, A.G. Suborder Adephaga, Polyphaga Incertae Sedis, Infraorder Staphyliniformia. In Mezozoiskie zhestkokryiye; Arnoldi, L.V.; Zherikhin, V.V.; Nikritin, L.M.; Ponomarenko, A.G., Eds. Trudy Paleontol. Inst. Akad. Nauk SSSR 1977, 161, 17-119.

142. Schimmel, R. Die Megapenthini-Arten Süd- und Südostasiens. Vierter Teil: Phylogenese, Zoogeographie und Katalog (Coleoptera: Elateridae). Pollichia Buch 2005, 48, 1-411.

(C) 2020 by the authors. Licensee MDPI, Basel, Switzerland. This article is an open access article distributed under the terms and conditions of the Creative Commons Attribution (CC BY) license (http://creativecommons.org/licenses/by/4.0/). 